\title{
The Tad Pilus Apparatus of 'Candidatus Liberibacter asiaticus' and Its Regulation by VisNR
}

\author{
Maxuel Andrade ${ }^{1}$ and Nian Wang ${ }^{1,2, \dagger}$ \\ ${ }^{1}$ Citrus Research and Education Center (CREC), Department of Microbiology and Cell Science, Institute of Food and \\ Agricultural Sciences, University of Florida, Lake Alfred, FL 33850, U.S.A. \\ ${ }^{2}$ China-USA Citrus Huanglongbing Joint Laboratory (A joint laboratory of the University of Florida Institute of Food and \\ Agricultural Sciences and Gannan Normal University), National Navel Orange Engineering Research Center, Gannan Normal \\ University, Ganzhou 341000, China
}

Accepted 29 March 2019.

Citrus huanglongbing (HLB) is one of the most destructive diseases affecting citrus plants. 'Candidatus Liberibacter asiaticus', an uncultivated $\alpha$-proteobacteria, is the most widely spread causal agent of HLB and is transmitted by the Asian citrus psyllid Diaphorina citri. ' $\mathrm{Ca}$. L. asiaticus' attachment to the psyllid midgut is believed to be critical to further infect other organs, including the salivary gland. In this study, the type IVc tight adherence (Tad) pilus locus encoded by ' $\mathrm{C} a$. L. asiaticus' was characterized. The Tad loci are conserved among members of Rhizobiaceae, including ' $\mathrm{Ca}$. $\mathrm{L}$. asiaticus' and Agrobacterium spp. Ectopic expression of the ' $\mathrm{Ca}$. $\mathrm{L}$. asiaticus' cpaF gene, an ATPase essential for the biogenesis and secretion of the Tad pilus, restored the adherence phenotype in cpaF mutant of A. tumefaciens, indicating $\mathrm{CpaF}$ of ' $\mathrm{Ca}$. L. asiaticus' was functional and critical for bacterial adherence mediated by Tad pilus. Quantitative reverse transcription PCR (qRT-PCR) analysis revealed that ' $\mathrm{C}$. $\mathrm{L}$. asiaticus' Tad pilus-encoding genes and ' $\mathrm{Ca}$. $\mathrm{L}$. asiaticus' pilin gene $f l p 3$ were upregulated in psyllids compared with in planta. A bacterial one-hybrid assay showed that ' $\mathrm{Ca}$. $\mathrm{L}$. asiaticus' VisN and VisR, members of the LuxR transcriptional factor family, were bound to the $f l p 3$ promoter. VisNR regulate $f l p 3$. Negative regulation of the flp3 promoter by both VisN and VisR was demonstrated using a shuttle strategy, with analysis of the phenotypes and immunoblotting together with quantification of the expression of the $f l p 3$ promoter fused to the $\beta$-galactosidase reporter gene. Comparative expression analysis confirmed that ' $\boldsymbol{C} a$. L. asiaticus' vis $N R$ was less expressed in the psyllid than in the plant host. Further, motility and biofilm phenotypes of the visNR mutant of $\mathrm{A}$. tumefaciens were fully complemented by expressing ' $\mathrm{Ca}$. $\mathrm{L}$. asiaticus' visNR together. The physical interaction between VisN and VisR was confirmed by pull-down and stability assays. The interaction of the flp3 promoter with VisR was verified by electrophoretic mobility shift assay. Taken together, the results revealed

${ }^{\dagger}$ Corresponding author: N. Wang; nianwang@ufl.edu

Funding: This study was supported by the U.S. Department of Agriculture National Institute of Food and Agriculture (grant number 2018-7001627412), Florida Citrus Initiative, and the Florida Citrus Research and Development Foundation.

*The e-Xtra logo stands for "electronic extra" and indicates that eight supplementary figures and two supplementary tables are published online.

The author(s) declare no conflict of interest.

() 2019 The American Phytopathological Society the contribution of the Tad pilus apparatus in the colonization of the insect vector by ' $\mathrm{Ca}$. $\mathrm{L}$. asiaticus' and shed light on the involvement of VisNR in regulation of the Tad locus.

Keywords: attachment, bacterial pathogenesis, citrus, greening, Liberibacter, pilus, regulation

Citrus huanglongbing (HLB), or citrus greening disease, is the most economically important disease affecting citrus production worldwide (Bendix and Lewis 2018; Clark et al. 2018; Li et al. 2019; Wang et al. 2017b). The most common causal agent associated with HLB, phloem-residing and vectorborne 'Candidatus Liberibacter asiaticus', has not yet been cultivated (Bendix and Lewis 2018; Coletta-Filho et al. 2014), which has hindered the understanding of its biology and virulence mechanism. 'Candidatus Liberibacter' spp. belong to the Rhizobiaceae family and cause diseases on many plant species. In addition to ' $\mathrm{Ca}$. L. asiaticus', ' $C a$. L. africanus' and ' $C a$. L. americanus' also cause citrus HLB (Duan et al. 2009; Kuykendall et al. 2012; Wulff et al. 2014). 'Ca. L. solanacearum' causes diseases on members of the Solanaceae family (e.g., "zebra chip" disease in potato) (Wang et al. 2017a and 2017b). 'Ca. L. asiaticus' is transmitted by the phloem-feeding insect vector Asian citrus psyllid, Diaphorina citri. $D$. citri acquires ' $C a$. L. asiaticus' from HLB-diseased plants as nymphs or adults, but the propagation of the pathogen will occur only after the colonization of the psyllid midgut by ' $\mathrm{Ca}$. L. asiaticus' (Ammar et al. 2016; Sétamou et al. 2016). It is assumed that ' $\mathrm{Ca}$. L. asiaticus' attachment to the psyllid midgut is critical to further infect other organs, including the salivary gland. Thus, understanding the ' $\mathrm{Ca}$. L. asiaticus' adherence mechanism to the psyllid midgut, as well as to other psyllid tissues, might provide useful information to prevent ' $\mathrm{Ca}$. L. asiaticus' transmission.

Comparative analysis of the genome sequences of ' $\mathrm{Candi}$ datus Liberibacter' spp. allowed a conserved gene cluster encoding the Tad (tight adherence) pilus apparatus (also termed type IVc pili) in their chromosomes to be identified (Duan et al. 2009; Ellison et al. 2017; Roux et al. 2012; Tomich et al. 2007). This gene cluster also is found in the genome of many bacterial species of Rhizobiaceae, including Sinorhizobium meliloti and Agrobacterium tumefaciens (Capela et al. 2001; Wang et al. 2014). Interestingly, two gene clusters encoding the Tad pilus apparatus are found in the $S$. meliloti genome, and these genes are required for efficient nodulation of the alfalfa host (Barnett et al. 2001; Capela et al. 2001; Zatakia et al. 2014). Type IV pili 
are widespread appendages whose assembly initiates in the bacterial inner membrane and expands out to the extracellular environment (Costa et al. 2016; Leighton et al. 2015). They participate in adhesion, cell-cell interactions, DNA transport, and motility (Mignolet et al. 2018). During the assembly process the type IV pilins are oligomerized through the secretion system apparatus with energy generated by the ATPases (Chung et al. 2014). Type IV pili are classified into three types-type IVa, IVb, and IVc-based on the phylogenetic analysis and features of their structural proteins and pilins (Ellison et al. 2017; Roux et al. 2012; Thompson et al. 2018). Pilins are processed before oligomerization by a prepilin peptidase, which is an inner membrane enzyme (de Bentzmann et al. 2006). The prepilin peptidase recognizes and cleaves the amino-terminal leader peptide, which is short (five to six residues) in type IVa pilins and longer (13 to 30 residues) in type IVb and IVc pilins (Ellison et al. 2017; O'Connell Motherway et al. 2011). The primary structure of the type IVc or Tad pilins shows distinct regions: a leader sequence in the amino terminal, a flp (fimbrial low-molecular weight protein) motif, hydrophobic region, and nonconserved carboxi terminal. Tad pilus apparatus is encoded by a limited number of genes clustered intro one or more operons that are likely acquired by horizontal transfer in some bacterial species (Angelov et al. 2015; Hansen and Forest 2006; Roux et al. 2012).

The Tad pili are usually not associated directly with motility. Instead, they are involved in adherence (e.g., the Cpa [Caulobacter pilus assembly] system in Caulobacter crescentus and Tad in Aggregatibacter actinomycetemcomitans) (Clock et al. 2008; Mignolet et al. 2018). For Pseudomonas aeruginosa, it has been demonstrated that the Tad pilus apparatus is required for bacterium-to-surface attachment, bacterium-to-bacterium contact and aggregation, and adhesion of bacterial cells to the host respiratory epithelial cells (Bernard et al. 2009). Interestingly, recent findings evidenced that the Tad pili apparatus in $C$. crescentus may sense the surface contact by resistance to pilus retraction, which is sufficient to stimulate holdfast synthesis that promotes the attachment to the surface (Ellison et al. 2017). In the early stage of Tad pili assembly, TadZ/CpaE functions as a hub for recruiting other pilus components (Tomich et al. 2007). In the current model of Tad pili biogenesis, the Flp pilins, TadE/CpaJ, and TadF/CpaK preproteins are targeted to the inner membrane, where they are processed by the TadV/CpaA prepilin peptidase. The TadE/CpaJ and TadF/CpaK pseudopilins may form an oligomeric structure in the periplasm, which guides pilus assembly and provides contact with the outer membrane complex. The TadD/CpaO and RcpB/CpaD proteins facilitate the assembly of the outer membrane RcpA/CpaC secretin complex. The TadA/CpaF ATPase provides energy for Flp pilin assembly into the filament, which is facilitated by the $\mathrm{TadB} / \mathrm{CpaG}$ and $\mathrm{TadC} / \mathrm{CpaH}$ inner membrane proteins. The TadG protein might serve as a membrane anchor for the Flp pilus. The Flp pilus is threaded through the $\mathrm{RcpA} / \mathrm{CpaC}$ secretin complex in the outer membrane, which is gated by $\mathrm{RcpB} / \mathrm{CpaD}$ protein (Mignolet et al. 2018; Roux et al. 2012; Tomich et al. 2007).

The signals that trigger the expression and assembly of the Tad pilus apparatus in each bacterium specie may be different. For example, in the bacterial predator Bdellovibrio bacteriovorus the tad pilus genes are upregulated in the initial stage of the predation process, and studies have shown they are essential for predation through attachment to the prey (Avidan et al. 2017). Perhaps the activation of the tad pilus genes in $B$. bacteriovorus is related to the presence of the prey in the environment. For the phytopathogen Pectobacterium atrosepticum, it was observed that the expression levels of the tad genes were increased under plant-mimicking conditions compared with rich growth medium (Nykyri et al. 2013).

In this study the Tad pilus of ' $\mathrm{Ca}$. L. asiaticus' is characterized. The data indicate that the Tad pilus of ' $\mathrm{Ca}$. L. asiaticus' is upregulated in psyllids and may contribute to the bacterial adherence and colonization of psyllid tissues and organs, such as the midgut. A. tumefaciens also was utilized as a model to reveal how the flp3 pilin gene is regulated by VisN and VisR of ' $\mathrm{Ca}$. L. asiaticus'. This study advances our understanding of ' $\mathrm{Ca}$. L. asiaticus' factors that are important for the colonization of psyllids and the spread of HLB disease and of how the ' $\mathrm{C} a$. L. asiaticus' attachment apparatus is regulated by VisNR.

\section{RESULTS}

\section{Type IVc Tad pilus locus is conserved} among 'Candidatus Liberibacter' spp.

Comparative genomic analyses showed that the Tad pilusencoding gene cluster is conserved in the 'Candidatus Liberibacter' spp. whose genome sequences are available in GenBank. Its structure is similar to the Tad loci of other Rhizobiaceae species (e.g., A. tumefaciens) (Fig. 1). The Tad gene clusters in 'Candidatus Liberibacter' spp. encode for all proteins that are necessary for assembly and function of the Tad pilus apparatus. Recently, the gene annotated as pilQ in the Tad cluster, due to its homology with the T2SS-T3SS Pil-N domain of the secretin protein $\mathrm{CpaC} / \mathrm{RpaA} / \mathrm{PilQ}$, was found to be important for Tad pilus assembly in $C$. crescentus and was reannotated as cpaI (Christen et al. 2016). Sequence alignment of CpaI homologs in liberibacters are shown in Supplementary Figure S1. The Tad pilin genes (Fig. 1) are multicopies from four to seven genes in the chromosomes of the 'Candidatus Liberibacter' spp., which indicates that duplication and divergence of $f l p$ genes may be important in the diversification of the colonization properties of these organisms (Avidan et al. 2017; Kachlany et al. 2001; Zatakia et al. 2014). The Flp pilins of 'Candidatus Liberibacter' spp. contain multiple conserved regions, a leader peptide sequence, a Flp motif, and a hydrophobic region. However, the carboxi-terminal region of the Flp pilins is not conserved, which may be important for the interaction of the "tip" of the Tad pilus with different surfaces (Roux et al. 2012). The comparative analysis of the primary structures of the seven pilins encoded by ' $\mathrm{Ca}$. L. asiaticus' is shown in Supplementary Figure S2.

\section{Ectopic expression of ' $\mathrm{Ca}$. L. asiaticus' cpaF gene restored the adherence phenotype in the cpaF mutant of $A$. tumefaciens.}

The $c p a F$ gene encodes for the ATPase of the Tad pilus and is essential for the biogenesis and secretion of the pilins (Tomich et al. 2007). CpaF shares 70 to $100 \%$ identity among 'Candidatus Liberibacter' spp. and other members of Rhizobiaceae, such as A. tumefaciens (Duan et al. 2009; Wang et al. 2014). In this study $A$. tumefaciens was used as a model to study the function of $\mathrm{CpaF}$ of ' $\mathrm{Ca}$. L. asiaticus' because it is not cultured in vitro. The cpaF gene of $A$. tumefaciens was inactivated by homologous recombination, and the cpaF deletion was confirmed by PCR (Fig. 2A). The $\triangle c p a F$ mutant strain showed a reduction of $50 \%$ in adherence to borosilicate glass tubes compared with the wild-type and complemented strains carrying 'Ca. L. asiaticus' cpaF or A. tumefaciens cpaF genes (Fig. 2B). The ectopic expression of $\mathrm{CpaF}$ in the complemented strains was confirmed by immunoblotting with antibodies against anti-FLAG Tag (Fig. 2C). The total protein extracts were verified using the loading control probed with anti-GroEL 
(Fig. 2C). These data showed that CpaF of ' $\mathrm{Ca}$. L. asiaticus' is functional and critical for bacterial adherence mediated by Tad pilus.

' $C a$. L. asiaticus' Tad pilus genes are highly expressed during colonization of psyllids.

To investigate the function of Tad pilus genes of ' $\mathrm{Ca}$. $\mathrm{L}$. asiaticus', their gene expression in planta and in psyllids was compared. The presence of ' $\mathrm{Ca}$. L. asiaticus' RNA in the leaf and psyllid RNA samples was verified by one-step reverse transcription PCR (RT-PCR) using the ' $\mathrm{Ca}$. L. asiaticus' specific primers Las16SF and Las16SR (Fig. 3A). qRT-PCR analyses showed that the expression of Tad pilus-encoding genes was higher in the insect host than in the plant host, ranging from a two- to 12 -fold increase (Fig. 3B). We also assessed the expression of the five genes encoding the Tad pilins that have a complete secretory signal (Fig. 3C and D). Interestingly, only the flp3 gene was highly induced in psyllids, whereas the four other flp genes were downregulated in psyllids. Altogether, the comparative gene expression studies suggested that the Tad pilus apparatus and the Flp3 pilin may play important roles during the colonization of the insect host by 'Ca. L. asiaticus'.

VisN and VisR, two LuxR transcriptional factors, regulate $f l p 3$ expression in ' $\mathrm{Ca}$. L. asiaticus'.

After determining that Flp3 pilin is highly expressed in psyllids, an attempt was made to identify which transcriptional factor of ' $\mathrm{Ca}$. L. asiaticus' regulates its expression. First, multiple regulators of ' $\mathrm{Ca}$. L . asiaticus' were tested using a bacterial one-hybrid assay. For this assay, the flp3 promoter region ( -30 to -280$)$ was fused with reporter gene his3 using the plasmid $\mathrm{pH} 3 \mathrm{U} 3$. Different ' $\mathrm{Ca}$. L. asiaticus' transcriptional factors were cloned downstream of the lacUV5 promoter and fused with the carboxi terminal of the Escherichia coli RNA polymerase omega subunit and FLAG Tag into the plasmid pB1H2 22 (Noyes et al. 2008). All of the constructs were used to transform the E. coli mutant strain ( $\Delta$ rpoZ:zeo, $\Delta$ hisB), and selection assays in minimal media without histidine plus 3 aminotriazole and antibiotics were performed (Noyes et al. 2008).

To test the feasibility of this assay, the first selection test was performed with the plasmids harboring, respectively, the promoter of the $l d t P$ gene and its transcriptional regulator $l d t R$ in ' $C a$. L. asiaticus', which were previously characterized (Pagliai et al. 2014 and 2017). The transactivation of the promoter $l d t P$ by LdtR was verified (Supplementary Fig. S3), indicating the feasibility of the bacterial one-hybrid assay.

The flp3 promoter was activated by in-trans expression of the full-length $v i s R$ or the carboxi terminal of both $v i s R$ and $v i s N$ that encode a DNA-binding domain helix-turn-helix motif (Fig. 4A). However, the expression of the full-length $v i s N$ did not activate the flp3 promoter under the tested conditions (Fig. 4B). VisN and VisR are two LuxR homologs encoded by several Rhizobiaceae species (Rotter et al. 2006; Sourjik et al. 2000; Xu et al. 2013). The expression of different ' $C a$. L. asiaticus' transcriptional regulators fused to the omega subunit in this assay was confirmed by immunoblotting (Fig. 4C). The transactivation assays indicated that VisN and VisR can bind to the flp3 promoter and regulate its expression.

Ectopic expression of ' $\mathrm{Ca}$. L. asiaticus' vis $\mathrm{N}$ and vis $R$ restores the phenotypes in the A. tumefaciens visNR mutant.

The visN and visR genes belong to one operon that is conserved among rhizobia bacteria. VisN and VisR act together to regulate the cellular motility, depending on flagellum, exopolysaccharide production, and biofilm formation (Hoang et al. 2008; Sourjik et al. 2000; Tambalo et al. 2010; Xu et al. 2013). The function of ' $C a$. L. asiaticus' visN and visR was investigated, using A. tumefaciens as a model, by testing whether ' $C a$. L. asiaticus' vis $N$ and visR could complement the

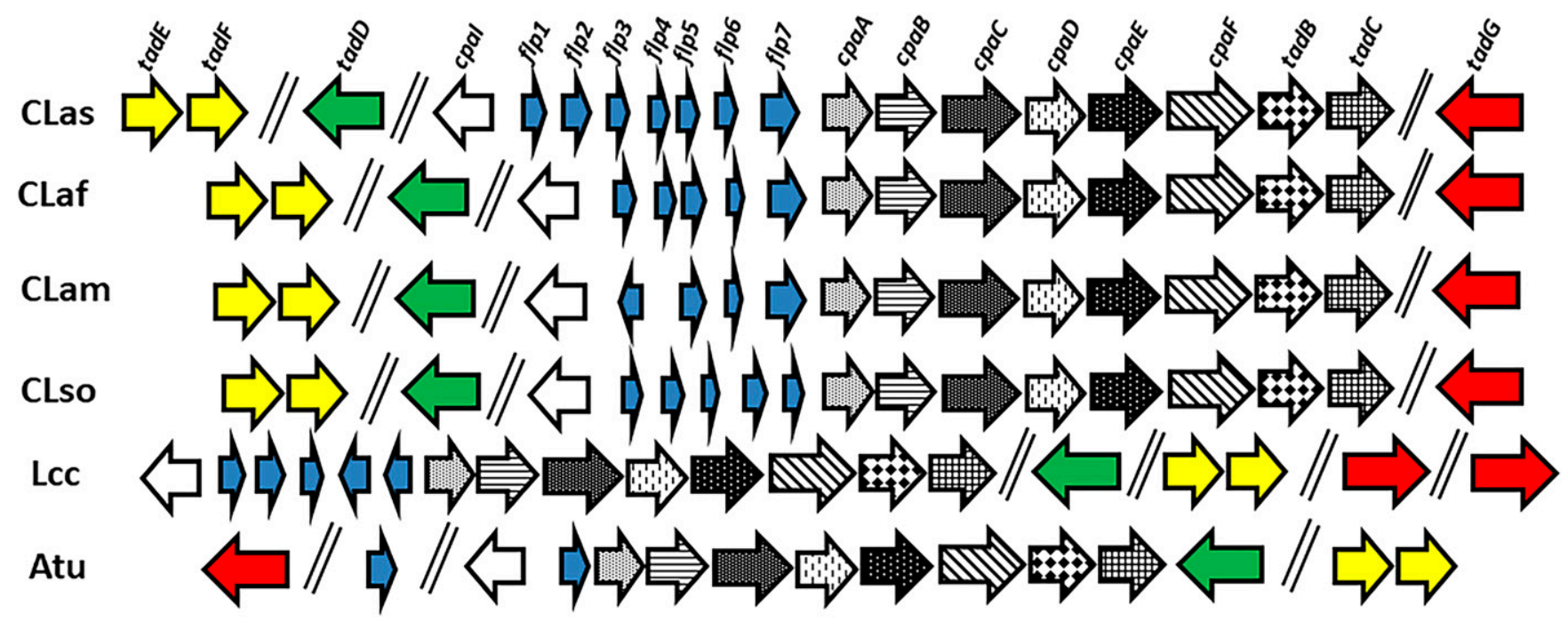

Fig. 1. Type IVc tight adherence (Tad) pilus-encoding gene cluster in 'Candidatus Liberibacter asiaticus'. Comparative genomics analysis revealed the presence of gene clusters encoding the Tad pilus in 'Candidatus Liberibacter' spp. The Tad pilus-encoding gene homologs in Agrobacterium tumefaciens C58 also are indicated. The gene cluster comparison was based on genomic and protein sequence analyses utilizing BLASTn and BLASTp (National Center for Biotechnology Information). Genes encoding for pseudopilins (tadE/cpaJ and tadF/cpaK); pilotin (tadD/cpaO); tadG, involved in stabilization of the pilus filament; cpaI; and Flp pilins are shown. Dotted, dashed, and shaded arrows represent essential genes for biogenesis of the Tad pilus apparatus that encodes the prepilin peptidase (cpaA), secretin (cpaC), periplasmatic subunits (cpaB and cpaD), inner membrane core ( $\operatorname{tadB} / \mathrm{cpaG}$ and tadC/cpaH), and ATPase (cpaF). CLas: 'Ca. L. asiaticus' (CP001677); CLaf: 'Ca. L. africanus' (CP004021); CLam: 'Ca. L. americanus' (CP006604); CLso: 'Ca. L. solanacearum' (CP002371); Lcc: Liberibacter crescens BT-1 (CP003789); Atu: A. tumefaciens C58 (CP014259). 
A. tumefaciens visNR mutant. Both vis $N$ and visR coding sequences in $A$. tumefaciens were deleted to produce the double-mutant $\Delta v i s N R$. The mutant strain was confirmed by PCR (Supplementary Fig. S4A). The swimming experiments exhibited loss of motility in the $\Delta v i s N R$ mutant strain or $\Delta v i s N R$ harboring constructs containing the A. tumefaciens vis $N$ or visR genes alone. The motility phenotype was restored by ectopic expression of $A$. tumefaciens visNR together, similar to previous studies (Xu et al. 2013). Likewise, the exopolysaccharide production, which was assessed using the polysaccharidebinding dye Congo red, and biofilm formation phenotypes were only fully restored to the wild-type traits by ectopic expression of both VisNR transcriptional regulators in the $A$. tumefaciens $\Delta v i s N R$ mutant strain. Taken together, these data indicate that in A. tumefaciens vis $N$ and visR work together to regulate motility, exopolysaccharide production, and biofilm formation. As previously shown, both VisN and VisR negatively regulated biofilm formation of $A$. tumefaciens on PVC 96-well microtiter plate surfaces (Fig. 5) (Xu et al. 2013). Surprisingly, both VisN and VisR positively regulated biofilm formation of $A$. tumefaciens in borosilicate glass tubes.

Next, the ability of ' $C a$. L. asiaticus' $v i s N$ and $v i s R$ to complement the A. tumefaciens $\Delta v i s N R$ double mutant was evaluated. Expression of ' $\mathrm{Ca}$. L. asiaticus' visN or visR alone partially restored swimming motility but did not recover exopolysaccharide production and biofilm formation on a PVC 96well microtiter plate surface in the A. tumefaciens $\Delta v i s N R$ mutant (Fig. 5A, 5B, and 5C). The phenotypes were fully restored in the A. tumefaciens $\triangle v i s N R$ mutant harboring both ' $C a$. L. asiaticus' visN and visR (Fig. 5A, 5B, and 5C). These results suggest that VisN and VisR homologs from A. tumefaciens and ' $C a$. L. asiaticus' work together to recognize and bind to the same target promoters in A. tumefaciens. Next, the ability of ' $C a$. L. asiaticus' VisN and VisR to positively regulate the expression of the ' $C a$. L. asiaticus' rem gene was tested. Rem, the regulator of exponential growth motility, is the master regulator of the flagellar genes and a participant in VisNR cascade in Rhizobiaceae (Rotter et al. 2006; Tambalo et al. 2010). The nonpolar mutant $\Delta r e m$ in A. tumefaciens was generated, and its impaired swimming motility was complemented by ectopic expression of ' $C a$. L. asiaticus' rem (Supplementary Fig. S5A). Expression of ' $\mathrm{Ca}$. L. asiaticus' rem was activated, and its proteins levels were significantly increased by ectopic expression of $A$. tumefaciens or ' $\mathrm{Ca}$. L. asiaticus' VisN and VisR together in the A. tumefaciens visNR mutant strain, but not by expression of VisN or VisR alone. Altogether, these data suggest that VisNR and Rem in ' $\mathrm{Ca}$. L. asiaticus' have a similar function to their counterparts in A. tumefaciens.

\section{Interaction between ' $\mathrm{Ca}$. L. asiaticus' VisN and VisR} increases VisN stability.

Genetics data suggest that VisN and VisR act together to control the same targets in rhizobia bacteria (Sourjik et al.
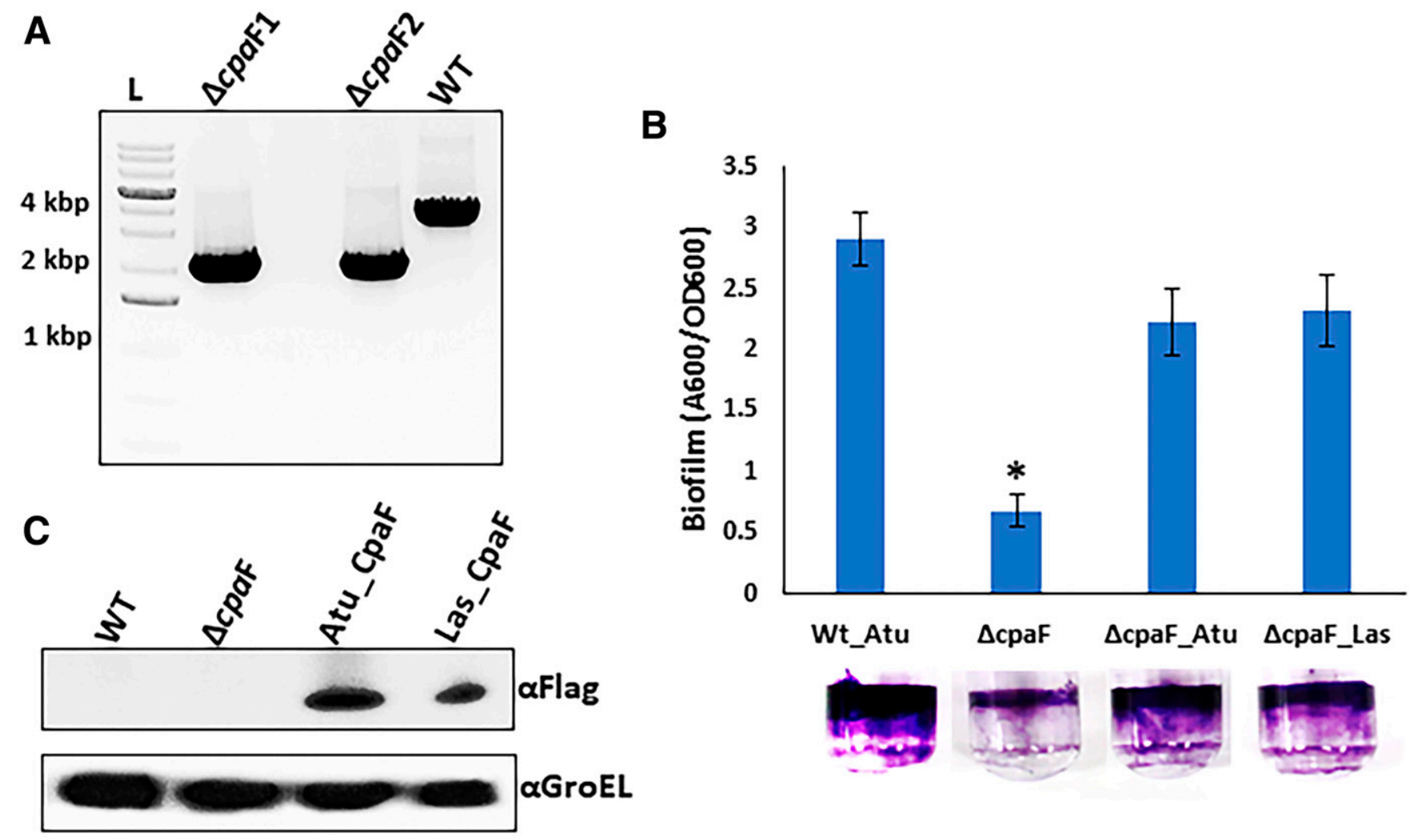

Fig. 2. Ectopic expression of the 'Candidatus Liberibacter asiaticus' cpaF gene restored adherence phenotype in the Agrobacterium tumefaciens cpaF mutant. A, Colony PCR confirmed the deletion of the cpaF gene in A. tumefaciens. $\Delta$ cpaF1 and $\Delta \mathrm{cpaF} 2$ represent independent mutant strains obtained by two steps of homologous recombination. $\Delta$ cpaF1 was used in the complementation assays. B, Quantitative biofilm assay in borosilicate glass tubes comparing the ability of the wild-type $A$. tumefaciens, $\Delta$ cpaF1 mutant, and complementation strains ( $\Delta$ cpaF_Atu and $\Delta$ cpaF_Las) to form biofilm. The biofilm assay was repeated three times, and means of four replicates and standard deviation values are indicated. The asterisk indicates a statistically significant difference $(P<0.05$ according to Student's $t$ test). C, Expression of Atu_CpaF and Las_CpaF in the complementation strains was verified by western blot. Anti-GroEL level was assessed as a loading control. M: DNA molecular marker; WT: A. tumefaciens wild-type strain; $\Delta \mathrm{cpaF} 1$ and $\Delta \mathrm{cpaF} 2$ : A. tumefaciens strains carrying an in-frame deletion within the $c p a F$ gene; $\Delta \mathrm{cpaF} \_$Atu and $\Delta \mathrm{cpaF} \_$Las: mutant $\Delta \mathrm{cpaF1}$ complemented with the $A$. tumefaciens $c p a F$ gene and with the 'Ca. L. asiaticus' cpaF gene, respectively; L: DNA ladder. 
2000; Tambalo et al. 2010; Xu et al. 2013). To test whether ' $C a$. L. asiaticus' VisN and VisR interact with each other, the pull-down assay was performed. It confirmed that ' $\mathrm{Ca}$. L. asiaticus' VisN and VisR form a protein heterocomplex. The constructs 6xHis-VisR and VisN-FLAG were expressed in $E$. coli, and the total protein extracts were used in the assay. Protein interactions were evaluated in Ni-NTA agarose beads (QIAGEN). The input and eluted proteins were analyzed by immunoblotting with anti-His Tag and anti-FLAG Tag antibodies (Fig. 6A).

The recombinant protein ' $\mathrm{Ca}$. L. asiaticus' VisN showed a lower amount of protein and was less soluble than ' $\mathrm{Ca}$. L. asiaticus' VisR when expressed in E. coli alone. It was hypothesized that VisR may be important for interacting with and assist VisN to fold correctly in the cell. Therefore, VisN was expected to be more resistant to degradation by proteases in the presence of VisR. To test this hypothesis, the stability or degradation ratio of the ' $\mathrm{Ca}$. L. asiaticus' VisN-FLAG expressed in the A. tumefaciens visNR double mutant, with and without ' $\mathrm{Ca}$. L. asiaticus' VisR, was checked at different time points after inhibiting the translation process with spectinomycin (Fig. 6B). The degradation of VisN-FLAG was significantly reduced in presence of VisR in the bacterial cell. Anti-GroEL levels were analyzed as a loading control (Fig. 6B).
Ectopic expression of ' $\mathrm{Ca}$. L. asiaticus' VisNR negatively regulates the expression of $f l p 3$ pilin.

To understand how VisN and VisR regulate the flp 3 promoter in vivo, the promoters of the three ' $\mathrm{Ca}$. L. asiaticus' genes $c p a A, c p a F$, and $f l p 3$ were fused to the $l a c Z$ reporter gene cloned in the pRA301 plasmid (Akakura and Winans 2002; Wang et al. 2014). The constructs were used to transform A. tumefaciens wild-type and $\Delta v i s N R$ mutant strains and evaluate the $\beta$-galactosidase activity under the tested growing conditions. The $\beta$-galactosidase activity of the $\Delta v i s N R$ mutant strain carrying the flp 3 promoter was about three times that in the wild type (Supplementary Fig. S6). However, there was no significant difference for the promoters of the cpaA and $c p a F$ genes between the wild-type and $\Delta$ visNR mutant strains. Accordingly, the results suggest that the LuxR-like proteins VisNR regulate the flp3 promoter.

Next, flp3 protein levels under the control of a constitutive promoter (trp) and its native promoter in A. tumefaciens wildtype, $\Delta v i s N R$, and complemented strains were analyzed. The expression of flp3 under control of the constitutive promoter was similar between wild-type and $\Delta v i s N R$ mutant strains (Fig. 7A). In the A. tumefaciens strains carrying flp 3 regulated by its native promoter, Flp3 protein levels were higher in the mutant strains $\triangle v i s N R$ and $\triangle v i s N R$ harboring either visN or visR than in the wild-type and $\Delta v i s N R$ strains carrying both visN and
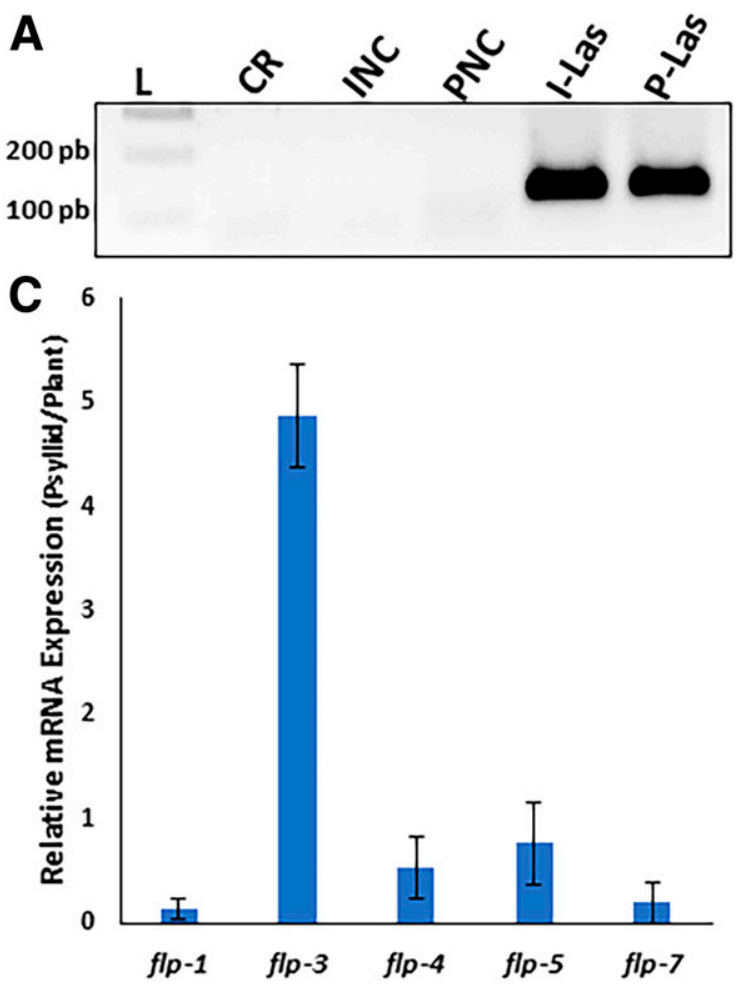

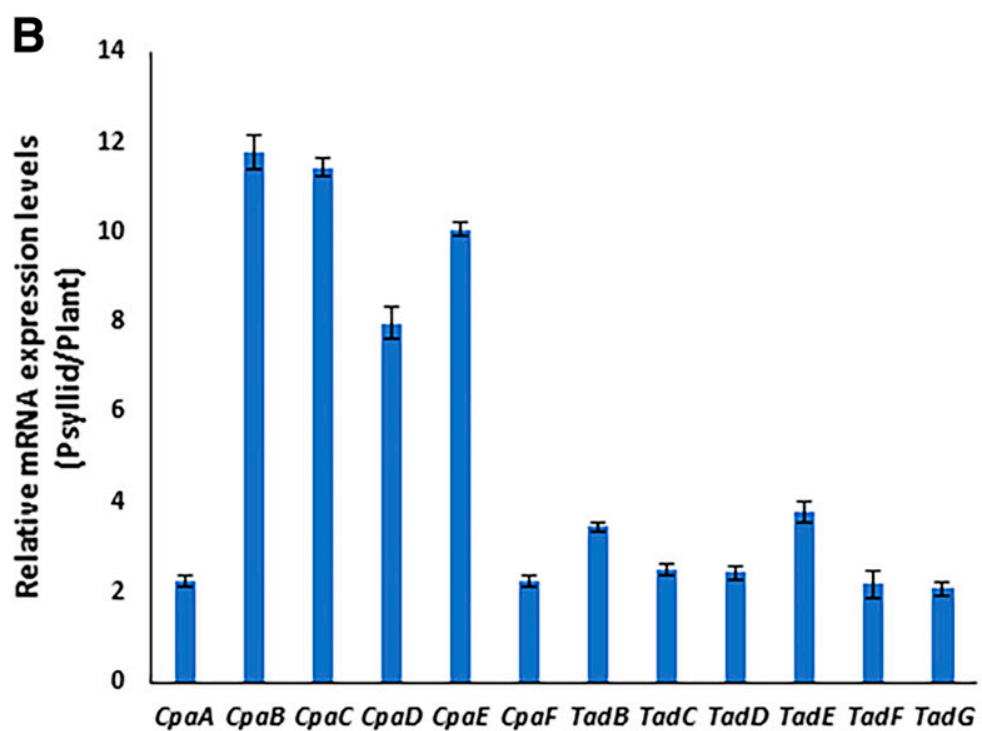

D

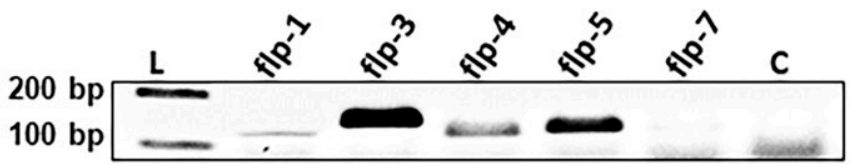

Fig. 3. Expression analysis of tight adherence (Tad) pilus-encoding genes in the psyllid and plant host infected with 'Candidatus L. asiaticus'. A, Reverse transcription PCR (RT-PCR) analysis to confirm infectivity of ' $\mathrm{Ca}$. L. asiaticus' in the insect vector (Diaphorina citri) and plant host (Citrus sinensis). RNA samples extracted from psyllids and sweet orange leaves (midrib and petiole) were subjected to one-step RT-PCR testing using Las16SF and Las16SR primers that specifically target the $16 \mathrm{~S}$ rRNA of ' $\mathrm{Ca}$. L. asiaticus'. PCR products were loaded and visualized in a $1.2 \%$ agarose gel. All samples, including positive and negative controls, were performed in triplicate. Only one representative result for each sample is shown. CR: negative control using water as template; INC and PNC: ' $C a$. L. asiaticus'-free psyllid and sweet orange plant, respectively; I-Las and P-Las: ' $C a$. L. asiaticus'-positive psyllid and sweet orange plant, respectively. $\mathbf{B}$ and $\mathbf{C}$, RNA samples from psyllids and plants infected with ' $\mathrm{Ca}$. L. asiaticus' were used to analyze the expression of the genes encoding structural Tad pilus components and genes encoding pilins ( $f l p 1$ through $f l p 7$ ), respectively, using one-step qRT-PCR. Statistical analysis of all data were conducted using Student's $t$ test $(P<0.05)$. Results represent the mean and standard deviation of three independent experiments, with four replicates each. D, RT-PCR products of the pilin-encoding genes ( $f l p 1$ through $f l p 7$ ) amplified from the infected psyllid RNA samples were loaded and separated in $1.2 \%$ agarose gel. L: DNA ladder; Flp: PCR products of the ' $\mathrm{Ca}$. L. asiaticus' genes coding fimbrial low-molecular weight protein (flp); C: 'Ca. L. asiaticus'-free psyllid as a negative control. 
visR (Fig. 7B). VisN and VisR of 'Ca. L. asiaticus' and A. tumefaciens were able to negatively control the expression of Flp3 together. These data suggest that during colonization of the insect vector ' $\mathrm{Ca}$. L. asiaticus' VisNR are downregulated or inhibited by unknown signal molecules to promote upregulation of the Flp3 pilin that is secreted by the Tad pilus apparatus. To test this hypothesis, a comparative analysis of gene expression in the psyllid and plant host was conducted, and ' $\mathrm{Ca}$. L. asiaticus' vis $N$ and visR were observed to be less expressed in psyllid (Supplementary Fig. S7), which corroborates findings from previous studies (Yan et al. 2013).

Transcriptomic studies of $A$. tumefaciens revealed several genes regulated by VisN and VisR, including rem and flagellar genes, but the promoter target sequences for VisN and VisR were not determined (Xu et al. 2013). Analysis of the 'Ca. L. asiaticus' rem and flp3 promoter sequences via alignment identified a conserved motif, 5'-GTWAATAWMRTTWATA- $3^{\prime}$, in the positions -127 to -112 and -96 to -81 , respectively. The results suggest ' $\mathrm{Ca}$. L. asiaticus' VisN and VisR act together to regulate Rem and Flp3 protein levels.

Physical interaction between VisR and the $f l p 3$ promoter.

The results suggest both ' $C a$. L. asiaticus' VisN and VisR regulate the flp3 promoter. The next goal was to provide experimental evidence for the physical interactions between the flp 3 promoter and VisN or VisR. Despite multiple attempts, the experiment failed to purify the ' $C a$. L. asiaticus' VisN recombinant protein or the complex VisNR protein in E. coli. Nonetheless, the recombinant ' $C a$. L. asiaticus' VisR expressed in $E$. coli was purified. The 6 HisVisR recombinant protein was purified by affinity chromatography and dialyzed against phosphate-buffered saline (PBS) buffer containing $2 \mathrm{mM}$ dithiothreitol (DTT) at $4^{\circ} \mathrm{C}$ overnight. The purified 6HisVisR protein samples were resolved in $12 \%$ sodium dodecyl sulfatepolyacrylamide gel electrophoresis gel (SDS-PAGE) and stained with Coomassie blue (Fig. 8A).

Physical interaction between the purified ' $\mathrm{Ca}$. L. asiaticus' 6 HisVisR and the flp 3 promoter region $(-198$ to +1$)$ was confirmed by electrophoretic motility shift assay (EMSA) (Fig. $8 \mathrm{~B}$ ), and no binding was observed when cpaA promoter was incubated with 6HisVisR (data not shown). Disruption of the binding of labeled flp3 probe with VisR by $10 \times$ and $100 \times$ unlabeled flp3 probe indicates the specificity of the interaction.

\section{DISCUSSION}

Liberibacter bacteria, including ' $\mathrm{Ca}$. L. asiaticus', form biofilm (Cicero et al. 2016 and 2017; Ghanim et al. 2016; Kruse et al. 2017; Wang and Trivedi 2013) inside the midgut lumen of
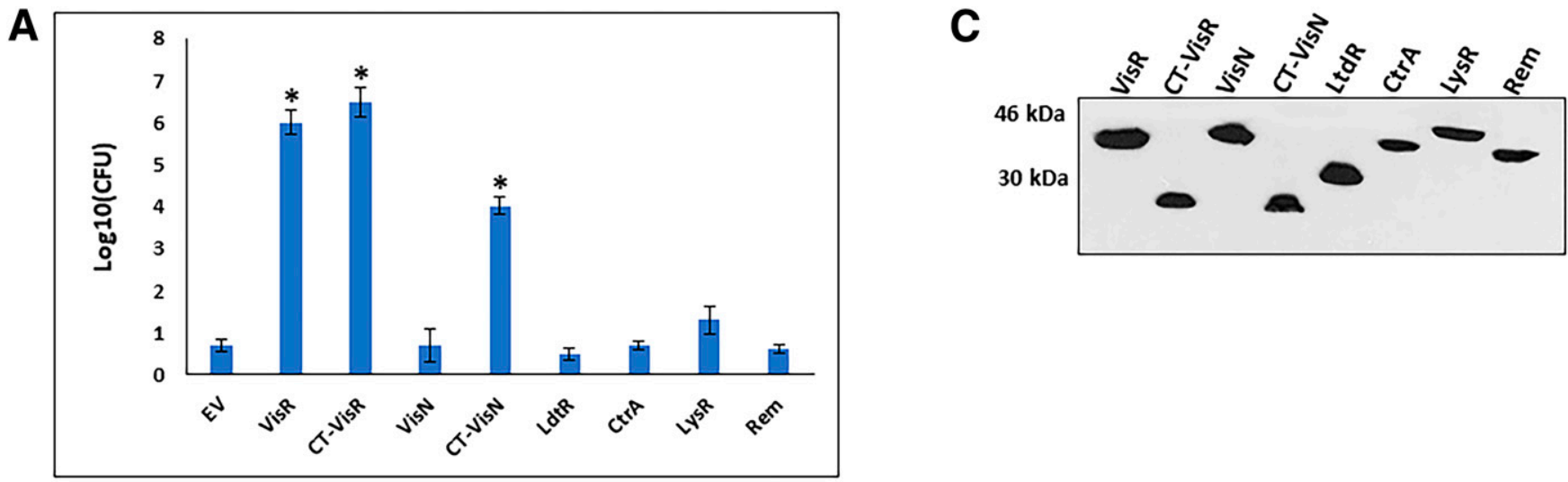

B
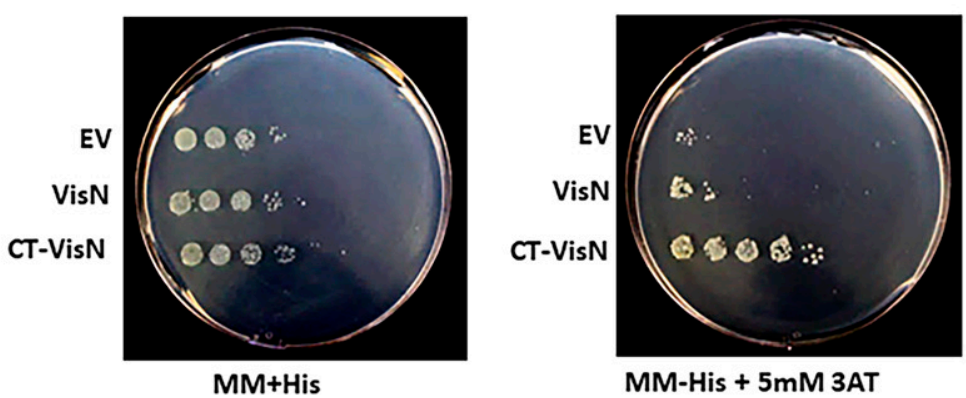

Fig. 4. The transcriptional regulators VisN and VisR bind to the promoter of flp3 that encodes the most abundant 'Candidatus L. asiaticus' pilin in infected psyllids. A, To evaluate the activation of the flp3 promoter fused to the reporter gene his 3 by ' $\mathrm{Ca}$. L. asiaticus' transcriptional factors, Escherichia coli cells carrying the constructs were plated in M9 minimal medium with $5 \mathrm{mM}$ 3-AT. The minimal medium with histidine served as a control in each assay. Cells harboring the flp3 promoter fused to his 3 were transformed with different ' $\mathrm{Ca}$. L. asiaticus' genes encoding transcriptional factors and plated in triplicate in the selective media. The mean and standard deviation colony counts are shown. Error bars represent the standard deviations $(n=3)$. The asterisk indicates a statistically significant difference $(P<0.05$, according to Student's $t$ test). B, A representative selection test is displayed with a control plate containing histidine and another plate without histidine plus $5 \mathrm{mM} \mathrm{3-AT}$. C, Expression of the ' $\mathrm{Ca}$. L. asiaticus' transcription factors under control of the Ptac promoter in transformed $E$. coli cells were confirmed by immunoblotting using anti-FLAG antibodies. EV: empty vector. VisR, VisN, LdtR, CtrA, LysR, and Rem are transcriptional factors coded by the ' $\mathrm{Ca}$. L. asiaticus' genome. CT-VisR and CT-VisN represent the carboxi-terminal region that encodes the DNA-binding domains of VisR and VisN, respectively. 
psyllids before moving through the midgut epithelial wall to enter the hemocoel and eventually infecting the salivary gland (Ghanim et al. 2016; Wang and Trivedi, 2013). Attachment is the initial step in biofilm formation. The Tad pilus apparatus was shown to be important for adherence of bacteria to host tissues during the colonization process (Kawahara et al. 2016; Li et al. 2012; Nykyri et al. 2013; O'Connell Motherway et al. 2011; Tomich et al. 2006; Wairuri et al. 2012). The involvement of Tad pilus in adherence of A. tumefaciens is supported by the data related to mutation of $c p a F$, an ATPase essential for the biogenesis and secretion of the type IVc pilus. Because of the high identity between the Tad loci of Agrobacterium spp. and ' $C a$. L. asiaticus' and complementation of the cpaF mutant of A. tumefaciens with ' $C a$. L. asiaticus' $c p a F$, we reasoned that the type IVc pilus might be involved in attachment to the epithelial cells of the psyllid midgut and to other tissues and organs inside the psyllid body. This is consistent with the higher expression level of Tad genes of ' $\mathrm{Ca}$. L. asiaticus' in psyllids compared with in planta. Some Tad genes (e.g., flp3) are found among ' $C a$. L. asiaticus' transcripts, with the highest abundance found in transcriptome analysis of the total RNA extracted from isolated bacteria colonizing the psyllid midgut (unpublished data). Additionally, it was shown that ' $\mathrm{Ca}$. L. solanacearum' produces pili- and flagella-like surface appendages and can form biofilm inside the potato psyllid (Bactericera cockerelli) (Cicero et al. 2016 and 2017). Tad genes are highly expressed in ' $\mathrm{Ca}$. L. solanacearum' haplotypes isolated from psyllids (Mendoza-Herrera et al. 2018). Liberibacter genomes encode for all of the genes needed to build a complete and functional Tad pili apparatus (Fig. 1), which might indicate that the Tad pili perform important functions in the adaptation of liberibacters to specific environments. Indeed, conservation of the Tad pilus-encoding loci among liberibacters supports the notion of a ubiquitous pili-mediated insect vector colonization mechanism for insectborne liberibacters.

The Tad genes were initially described for the periodontal pathogen Aggregatibacter actinomycetemcomitans (Tomich et al. 2007). Transcriptome analysis of the Bifidobacterium breve in a murine colonization model revealed differential expression of Tad pilus-encoding genes. Mutational analysis demonstrated that the tad gene cluster is essential for efficient in vivo murine gut colonization (O'Connell Motherway et al. 2011). Further, mutational analysis in Pectobacterium spp. demonstrated that the Tad pilus apparatus had a significant impact on the maceration ability of the pathogen in potato tubers, indicating that the Tad pilus-encoding gene cluster represents a novel virulence determinant in Pectobacterium spp. (Nykyri et al. 2013). Likewise, Tad pili were shown to be essential for the pathogenicity of Ralstonia solanacearum, as the tadA2 mutant was impaired in its ability to cause wilting of potato plants. In Rhizobiaceae, the Tad pilus apparatus was shown to increase the initial adherence of the bacterial cell to surfaces and to the host tissue (Thompson et al. 2018; Wang et al. 2014; Zatakia et al. 2014). Because ' $C a$. L. asiaticus' has

\section{A}
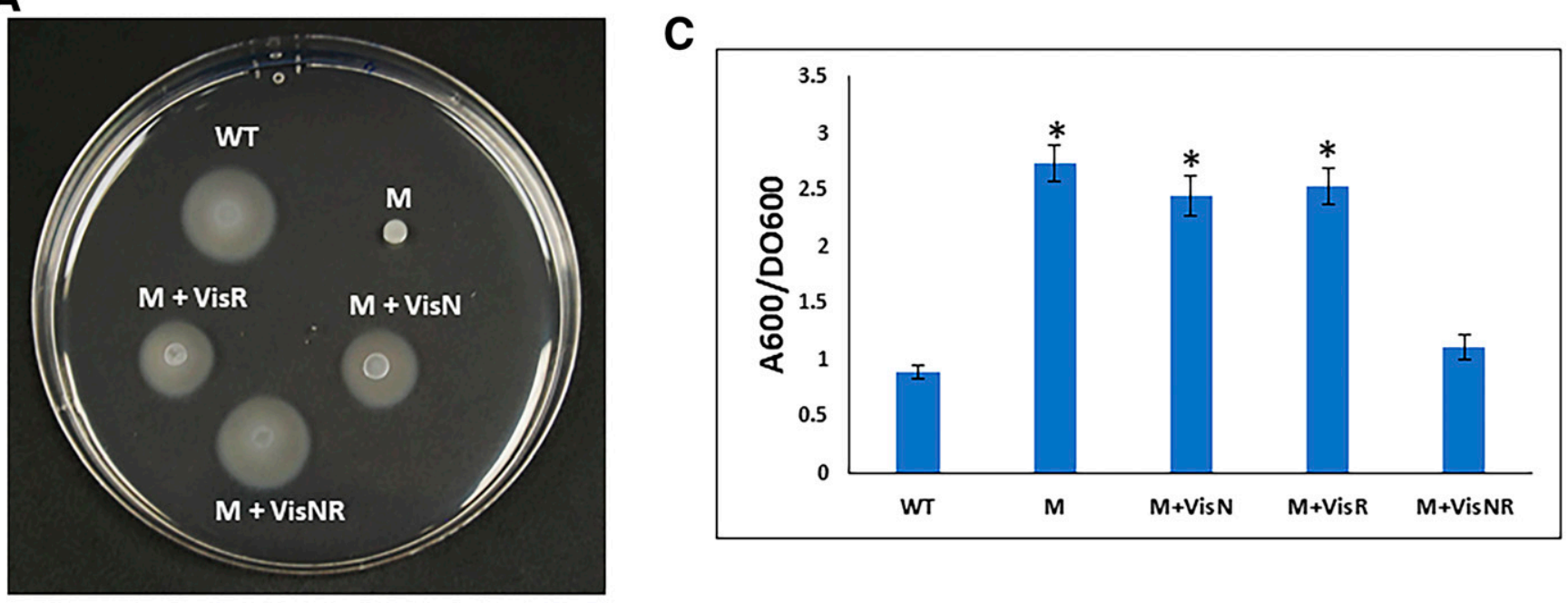

B

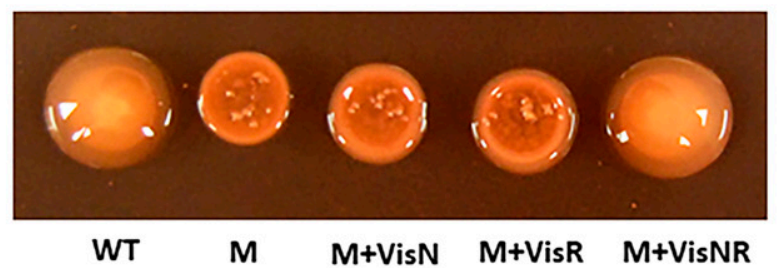

Fig. 5. Ectopic expression of 'Candidatus L. asiaticus' visNR homologs restores motility, exopolysaccharide production, and biofilm formation in the visNR double mutant of Agrobacterium tumefaciens. A, Effect of ectopic expression of ' $\mathrm{Ca}$. L. asiaticus' visN or visR alone and visNR together on the swimming motility of the A. tumefaciens visNR double mutant. B, Exopolysaccharide production in A. tumefaciens strains was evaluated by growing the cells in modified ATGN Congo Red media. C, Biofilm formation of the A. tumefaciens wild-type, visNR mutant and complemented strains was assessed in PVC 96-well microtiter plates at $28^{\circ} \mathrm{C}$ for $48 \mathrm{~h}$. Mean values plus/minus standard deviations $(n=4)$ are plotted. Mean values were compared with the wild type; asterisks indicate statistically significant differences $(P<0.05$, according to Student's $t$ test). WT: A. tumefaciens wild-type strain carrying the empty vector; M: doublemutant visNR of A. tumefaciens carrying the empty vector; M+VisR, VisN, and VisNR: double-mutant visNR of A. tumefaciens carrying the 'Ca. L. asiaticus' $v i s R$, vis $N$, and visNR gene, respectively. 
not been cultured in vitro, we are unable to provide direct genetic evidence regarding the function of Tad genes. Using A. tumefaciens as a model, we are able to show that some of the Tad genes are functional. For example, cpaF is functional in restoring the adherence phenotype of the cpaF mutant of A. tumefaciens.

The data revealed that in ' $\mathrm{Ca}$. L. asiaticus' the expression of the flp 3 gene is under the control of the two LuxR-type transcriptional regulators, VisN and VisR. visN and visR genes are localized in the same operon and conserved among members of the Rhizobiaceae. In $S$. meliloti in-frame deletions of $v i s N$ and vis $R$ resulted in mutant strains that were nonmotile and nonflagellated due to impaired flagellar synthesis (Sourjik et al. 2000). Complementation by expression of the homologous wild-type alleles completely restored swimming and swarming proficiency in both mutant strains. The data clearly suggest that VisN and VisR act in trans and are both required for flagellar synthesis (Sourjik et al. 2000). Furthermore, A. tumefaciens produces a unipolar polysaccharide (UPP) adhesin at cell poles that contact surfaces. Transposon mutagenesis screening identified visN and visR as UPP-negative regulators with hyperadherent phenotype. Likewise, as shown in $S$. meliloti, vis $N$ and $v i s R$ are activators of flagellar motility in A. tumefaciens, in addition to inhibiting UPP and cellulose production (Xu et al. 2013). In this study, we advanced our understanding of the regulatory roles of VisN and VisR by showing that they negatively regulate the flp3 gene, the pilin gene with the highest expression in psyllids among the seven ' $\mathrm{Ca}$. L. asiaticus' pilin genes. Inversely, 'Ca. L. asiaticus' vis $N$ and visR expression was shown to be downregulated in psyllids. Regulation of flp3 by VisNR might allow ' $C a$. L. asiaticus' to switch between forming biofilm and circulative status inside the psyllid hemocoel to facilitate its infection and transmission, considering the role of pilin in adherence. Indeed, a similar connection between Tad pilus function and differential expression of $f l p$ pilins was reported in previous studies for other bacteria species (Avidan et al. 2017; Kachlany et al. 2001; Zatakia et al. 2014). VisN and VisR share similarities within their C-terminal regions with the DNA-binding domains of LuxR and FixJ homologs, as well as to each other. However, the $\mathrm{N}$ termini of VisN and VisR do not resemble either the acyl-homoserine lactone-binding motif of LuxR homologs nor the phosphatereceiver domain of FixJ. The $\mathrm{N}$ termini of VisN and VisR also do not resemble each other. Indeed, the DNA-binding domain encoded by the carboxi-terminal region of VisN and VisR in A. tumefaciens and ' $\mathrm{Ca}$. L. asiaticus' showed high identity of more than $80 \%$. In contrast, the amino terminal of the LuxR homolog proteins VisN and VisR are less conserved, with about $40 \%$ of identity. Thus, the $\mathrm{N}$ termini of $A$. tumefaciens and ' $\mathrm{Ca}$. L. asiaticus' VisN and VisR regulators might bind to different
A

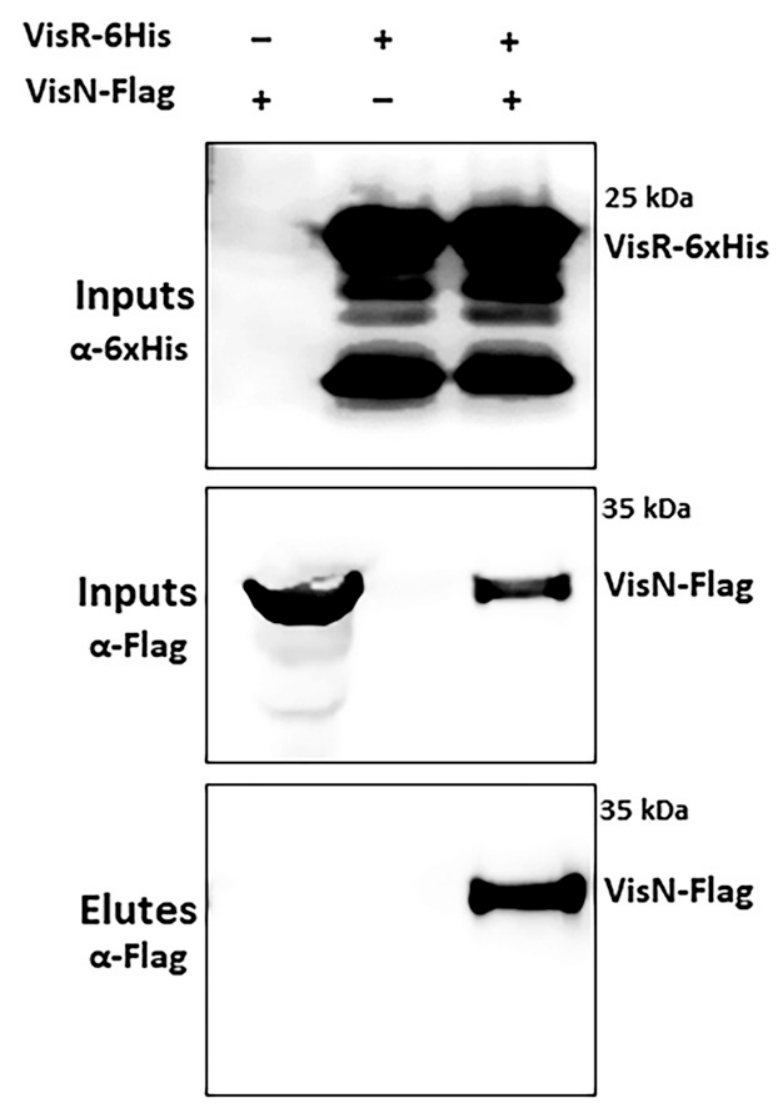

B
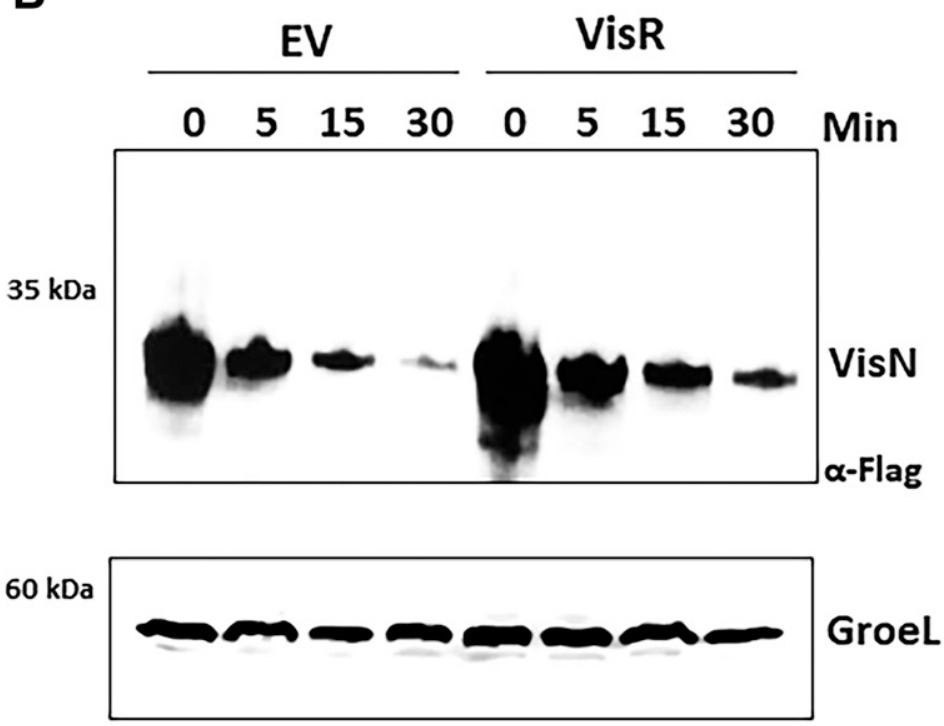

Fig. 6. Pull-down and stability assays show a physical interaction between 'Candidatus L. asiaticus' VisN and VisR. A, The protein 6xHisVisR was used as bait for the pull-down assay. Protein 6xHisVisR was expressed in Escherichia coli, immobilized and washed on nickel-IMAC beads (Thermo Fisher Scientific), and incubated with $E$. coli lysates containing 4xFLAG-VisN. Total cell extracts (inputs) and eluted protein (elutes) were immunoblotted using anti-FLAG and anti6xHis antibodies. B, Stability of 'Ca. L. asiaticus' VisN was increased when coexpressed with VisR. The Agrobacterium tumefaciens visNR double-mutant strain harboring ' $\mathrm{Ca}$. L. asiaticus' vis $N$ was cotransformed with empty plasmid pBKT or pBKT-visR. Cells of both strains were cultured, and protein synthesis was blocked by addition of spectinomycin at $1 \mathrm{mg} / \mathrm{ml}$ into bacterial culture. Samples collected at the indicated time were analyzed by immunoblotting. AntiGroEL antibody was used to normalize the amount of protein loaded. EV: A. tumefaciens double-mutant visNR carrying 'Ca. L. asiaticus' visN and pBKT empty plasmid; VisR: mutant visNR carrying both visN and visR genes of ' $\mathrm{Ca}$. L. asiaticus'. 
signal molecules. We cannot rule out the possibility that the upregulation of flp3 in psyllids is not only due to downregulation of ' $\mathrm{Ca}$. L. asiaticus' visN and visR gene expression but also to other mechanisms, such as the binding of specific molecules to the amino terminal of both proteins, which may regulate their function.

In conclusion, we have shown that ' $\mathrm{Ca}$. L. asiaticus' contains a functional Tad pili that may be involved in attachment to and colonizing of psyllid tissues and organs such as the midgut. VisN and VisR act together to negatively regulate the flp 3 gene by binding to its promoter region. Inhibition of ' $\mathrm{Ca}$. L. asiaticus' attachment to the epithelial cells in the psyllid midgut and to other psyllid tissues via Tad pilus may present a new path for reducing the colonization and transmission of ' $\mathrm{Ca}$. L. asiaticus' by the psyllid vector.

\section{MATERIALS AND METHODS}

Bacterial strains, plasmids, and growth conditions.

The bacterial strains, plasmids, and oligonucleotides used in this study are listed in Supplementary Tables S1 and S2. E. coli cells were grown at $37^{\circ} \mathrm{C}$ in Luria-Bertani (LB) medium ( $1 \%$ tryptone [wt/vol], $0.5 \%$ yeast extract [wt/vol], $1 \%$ sodium chloride [wt/vol], $\mathrm{pH} 7.5)$. A. tumefaciens wild-type strain C58 and the mutant strains were grown in $2 \times \mathrm{TY}$ medium $(1.6 \%$ tryptone [wt/vol], $1.0 \%$ yeast extract [wt/vol], $0.5 \%$ sodium chloride [wt/vol], $\mathrm{pH} 7.5)$ or ATGN medium (79 $\mathrm{mM} \mathrm{KH}_{2} \mathrm{PO}_{4}$, $15 \mathrm{mM}\left(\mathrm{NH}_{4}\right)_{2} \mathrm{SO}_{4}, 5 \mathrm{mM} \mathrm{MgSO} \cdot 7 \mathrm{H}_{2} \mathrm{O}, 1 \mathrm{mM} \mathrm{CaCl} 2$, $0.0071 \mathrm{mM} \mathrm{MnSO} \cdot 7 \mathrm{H}_{2} \mathrm{O}, 0.125 \mathrm{mM} \mathrm{FeSO} \cdot 7 \mathrm{H}_{2} \mathrm{O}, 0.5 \%$ glucose [wt/vol], $\mathrm{pH} 7$ ) with or without $1.5 \%$ (wt/vol) agar at $28^{\circ} \mathrm{C}$ (Morton and Fuqua 2012; Wise et al. 2006). For $s a c B$ counter-selection, 5\% sucrose was added to LB medium. Plasmids were introduced into $E$. coli by heat-shock at $42^{\circ} \mathrm{C}$ and into A. tumefaciens by electroporation (Weigel and Glazebrook 2006). For plasmid selection, the antibiotics were used at the following concentrations: kanamycin at $50 \mathrm{mg} / \mathrm{ml}$; ampicillin at $100 \mathrm{mg} / \mathrm{ml}$; spectinomycin at $50 \mathrm{mg} / \mathrm{ml}$; and gentamicin at $10 \mathrm{mg} / \mathrm{ml}$. Isopropyl- $\beta$-D-thiogalactopyranoside (IPTG) was used when necessary. For Congo red plates, the dye was dissolved in methanol at $20 \mathrm{mg} \mathrm{ml}^{-1}$. Filtered Congo red dye was added to ATGN medium (final concentration approximately $80 \mu \mathrm{g} \mathrm{ml}^{-1}$ ).
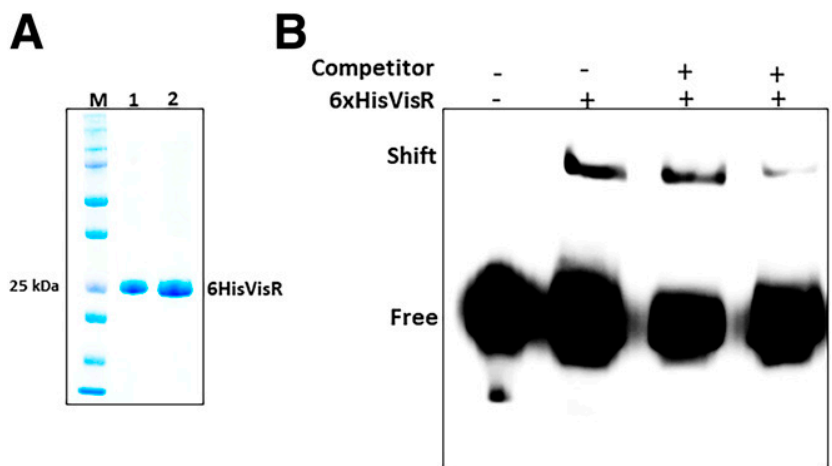

Fig. 8. VisR binds to the promoter region of 'Candidatus L. asiaticus' flp3. A, Purified 6HisVisR protein samples were resolved in $12 \%$ sodium dodecyl sulfate-polyacrylamide gel electrophoresis gel and stained with Coomassie blue. B, Electrophoretic mobility shift assay of $6 \mathrm{HisVisR}$ incubated with the biotin-labeled flp3 promoter fragment. From left to right: lane 1, free flp3 probe; lane 2, probe with purified 6HisVisR; lane 3, probe with 6 His VisR and $10 \times$ nonbiotinylated flp3 promoter; lane 4 , probe with 6 HisVisR and competitor $100 \times$ nonbiotinylated flp3 promoter. Shift: the complex formed by $6 \mathrm{His} V i s \mathrm{R}$ and biotinylated flp 3 promoter.

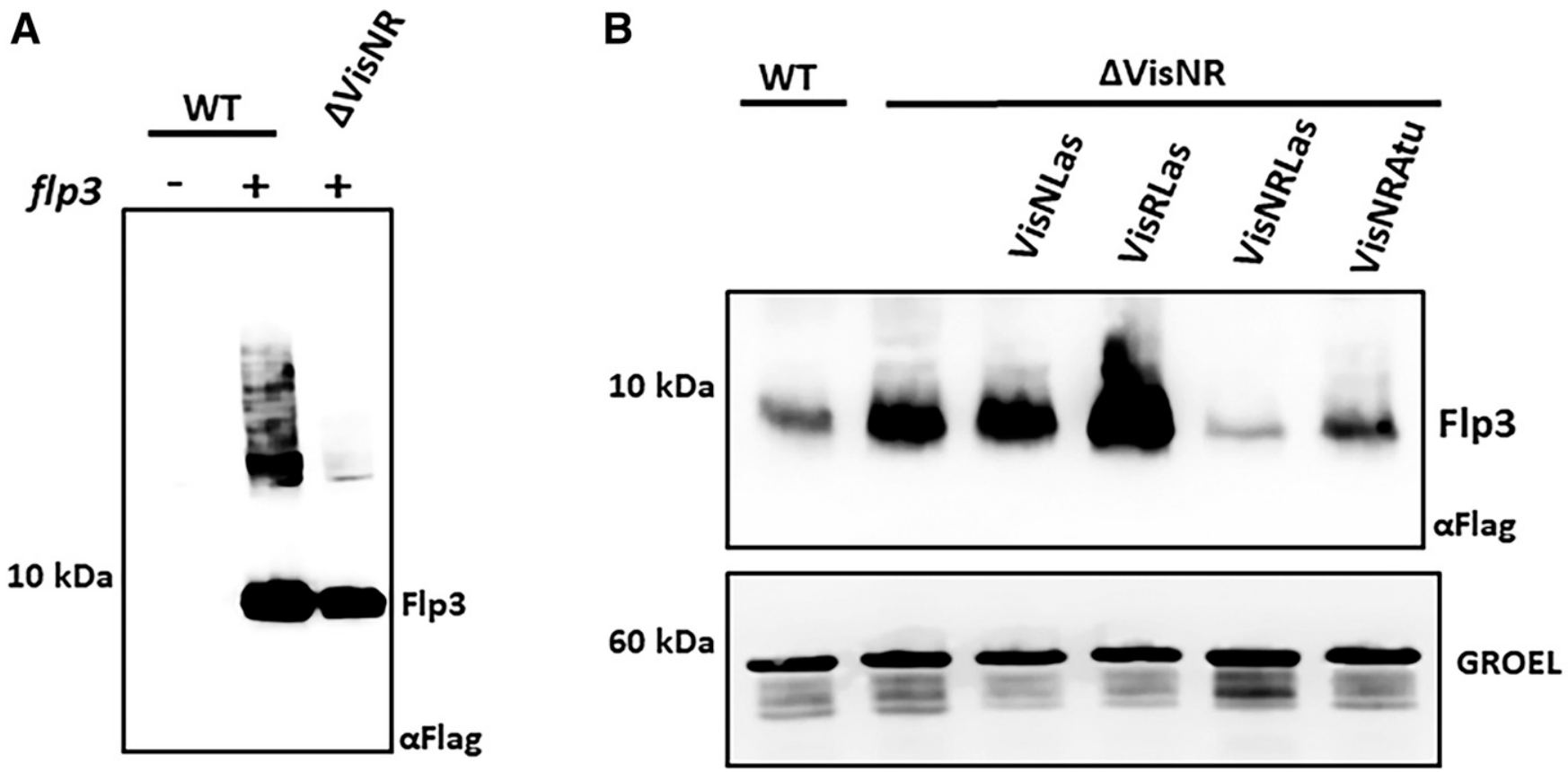

Fig. 7. VisN and VisR regulate the expression of 'Candidatus L. asiaticus' flp3. A, Ectopic expression of flp3 under control of the constitutive promoter Ptrc. Cells of Agrobacterium tumefaciens wild-type and visNR mutant strains harboring the flp3 coding region fused to Ptrc were grown to an optical density (OD) at $600 \mathrm{~nm}=1$ on modified ATGN medium and collected. Total cell extracts were subjected to immunoblotting. B, ' $C a$. L. asiaticus' VisN and VisR regulate the expression of flp3. A. tumefaciens wild-type and $\Delta v i s N R$ mutant strains carrying the plasmidborne ' $C a$. L. asiaticus' flp3 gene were cotransformed with ' $C a$. L. asiaticus' visN, visR, and visNR or A. tumefaciens visNR genes as indicated. Bacterial cells grown in modified $\mathrm{ATGN}_{\text {medium }}$ were collected at $\mathrm{OD}_{600}=1$. Protein extracts were resolved by sodium dodecyl sulfate-polyacrylamide gel electrophoresis and transferred to nitrocellulose. The blots were probed with antiFLAG antibodies. Anti-GroEL antibody was used to normalize the amount of protein loaded. Protein A conjugated with horseradish peroxidase was used to detect the antibody. 
Construction of the A. tumefaciens deletion mutants and complemented strains.

DNA manipulations and PCR were performed according to standard procedures (Sambrook and Russell 2006). Restriction digestion and DNA ligation were performed in accordance with the manufacturer instructions (New England Biolabs). To construct the cpaF (Atu0218), rem (Atu0573), and visNR (Atu0524-Atu0525) deletion mutants, approximately $1 \mathrm{~kb}$ of the upstream and downstream regions of the target genes was amplified by PCR from $A$. tumefaciens genomic DNA, and the two fragments were ligated to produce an in-frame deletion (Andrade et al. 2014). The generated fragments were then cloned into the EcoRI or HindIII restriction site of the pNPTS138 suicide vector, thus generating the plasmids pNPTS-cpaF, pNPTS-rem, and pNPTS-VisNR. These vectors were introduced into A. tumefaciens by electroporation, and the wild-type copy was replaced by the deleted version of each construct after two recombination events, as described previously (Andrade et al. 2014). To complement the generated knockout mutants, fragments including the deleted genes and approximately $500 \mathrm{bp}$ of upstream sequence were amplified by PCR from A. tumefaciens genomic DNA and inserted into the pBBR1-MCS-2 vector at the KpnI and XhoI restriction sites, or the deleted coding region was fused to lac promoter in pBBR1MCS-2. To verify whether the homologs encoded by ' $C a$. L. asiaticus' could complement the $A$. tumefaciens deleted mutant strains, the coding region of the genes CLIBASIA_03055 (cpaF), CLIBASIA_02065 (rem), and CLIBASIA_02905 and CLIBASIA_02900 (visN and visR, respectively) were amplified by PCR from ' $\mathrm{Ca}$. L. asiaticus' genomic DNA fused to lac or trp promoters and inserted into pBBR1-MCS-2 or pTF53. The genomic DNA for ' $C a$. L. asiaticus' was extracted from ' $C a$. L. asiaticus'-infected citrus grown in the psyllid room at the Citrus Research and Education Center (CREC, University of Florida, Lake Alfred, U.S.A.). The ' $C a$. L. asiaticus' genomic DNA was extracted using the Wizard DNA Genomic Purification Kit (Promega) following the manufacturer suggestions. The constructed plasmids were then transferred to the respective A. tumefaciens mutant strains by electroporation and selection for gentamicin or kanamycin resistance. All constructs were sequenced for confirmation.

\section{Biofilm, exopolysaccharide, and motility assays.}

Biofilm formation on glass surfaces and PVC 96-well microtiter plates was examined as described previously (Hibbing and Fuqua 2011; Li and Wang 2011), with modifications. The wild-type, mutant, and complemented cells were grown in $2 \times \mathrm{TY}$ medium overnight at $28^{\circ} \mathrm{C}$ with shaking $(180 \mathrm{rpm})$. Bacterial cells were pelleted and resuspended in ATGN medium to an optical density at $600 \mathrm{~nm}\left(\mathrm{OD}_{600}\right)$ adjusted to 0.05 (UV/Vis Spectrometer Lambda Bio, PerkinElmer). A 200- $\mu \mathrm{L}$ portion (1.5-ml volume) of adjusted bacteria was placed in the glass tubes and plate wells, respectively, and incubated at $28^{\circ} \mathrm{C}$ without agitation. After 2 days, bacterial growth was checked by measuring the $\mathrm{OD}_{600}$, and the culture was removed using a pipette. After repetitive water washes, the adhered cells were stained with $0.1 \%$ crystal violet $(\mathrm{CV})(\mathrm{wt} / \mathrm{vol})$ for $30 \mathrm{~min}$ at room temperature. After staining by $\mathrm{CV}$, the unbound stain was removed by water washing, and the $\mathrm{CV}$-stained cells were solubilized in $30 \%$ acetic acid and quantified by spectrophotometry at $600 \mathrm{~nm}$. Ratio $A_{600} / \mathrm{OD}_{600}$ represents the crystal violet quantification normalized by culture OD. The means of four replicates and standard deviation values are indicated. The motility assays were performed as previously described (Xu et al. 2013), with modifications. Bacteria were grown overnight in LB agar medium and bacterial cells were spotted on a plate containing ATGN medium plus 0.3\% agar (wt/vol) (Difco).
Plates were incubated at $28^{\circ} \mathrm{C}$ for $48 \mathrm{~h}$. The experiments were repeated three times, with three replicates each time. The exopolysaccharide production assay was conducted as described by Xu et al. (2013), with modifications. Bacterial cells were grown in $\mathrm{LB}$ medium overnight at $28^{\circ} \mathrm{C}$ and $180 \mathrm{rpm}$. The $\mathrm{OD}_{600}$ was adjusted to 0.1 (UV/Vis Spectrometer Lambda Bio), and $3 \mu \mathrm{L}$ of each strain was spotted on ATGN medium $1.5 \%$ agar (wt/vol) plus $2 \%$ glucose and Congo red dye (final concentration approximately $80 \mu \mathrm{g} \mathrm{ml}^{-1}$ ). Plates were incubated at $28^{\circ} \mathrm{C}$ for 3 days.

\section{RNA extraction and expression analysis of Tad pilus-encoding genes in infected citrus and psyllids by qRT-PCR.}

Psyllid colonies were maintained on ' $C a$. L. asiaticus'infected sweet orange ('Valencia') in a secured greenhouse with a temperature of $25 \pm 3^{\circ} \mathrm{C}$ and relative humidity of $50 \% \pm 5 \%$. The ' $C a$. L. asiaticus' status of the insects and citrus plants was verified by qPCR (Yan et al. 2013). Total RNA was extracted using the RNeasy Mini Kit (QIAGEN), according to the manufacturer instructions. For the extraction of RNA from ' $\mathrm{Ca}$. L. asiaticus'-infected plants, the midrib and petiole of symptomatic leaves were collected and ground in liquid nitrogen to a fine powder before RNA isolation. For RNA isolation from putative ' $C a$. L. asiaticus'-infected psyllids, 16 psyllids were pooled and ground in liquid nitrogen before RNA isolation. Purified RNA was treated with DNAse I-Free (QIAGEN), and successful removal of genomic DNA was confirmed by PCR using specific primers that annealed only to the CLIBASIA_03015 promoter region. RNA quantity was determined on a ND-8000 Nanodrop spectrophotometer (NanoDrop Technologies). Isolated plant and psyllid RNA samples were subjected to one-step qRT-PCR assay to test for infection by ' $\mathrm{Ca}$. L. asiaticus' using primers Las16SF-Las16SR, which specifically target the 16S rRNA of 'Ca. L. asiaticus' (Yan et al. 2013). Positive psyllid RNA samples for ' $C a$. L. asiaticus' were identified for further analysis of gene expression of the Tad pilus-encoding genes. All qRT-PCR analyses were performed using an Applied Biosystems 7500 Fast Real-time PCR system with a QuantiTect SYBR Green one-step RT-PCR kit (QIAGEN), as described by Yan et al. (2013). Relative expression levels of the Tad pilusencoding genes, which were normalized using the reference gene gyrB, were calculated with the $\Delta \Delta \mathrm{Ct}$ method (Livak and Schmittgen 2001). Statistical analyses of all data were conducted by Student's $t$ test $(P<0.05)$. Mean values derived from three independent experiments with four replicates each are shown. Primers were designed using Primer Express Software (Applied Biosystems).

\section{Immunoblotting.}

To analyze the specific protein levels in $E$. coli and A. tumefaciens strains, cells were grown in $2 \times \mathrm{TY}$ or AT minimal media to an $\mathrm{OD}_{600}$ of 0.5 . Bacterial cells were harvested, and pellets were resuspended in $50 \mathrm{mM}$ Tris- $\mathrm{HCl}(\mathrm{pH} 8.0)$, $100 \mathrm{mM} \mathrm{NaCl}, 0.1 \%$ Tween 20 , and $1 \mathrm{mg}$ of lysozyme per milliliter. Cells were ruptured by sonication. Cell-free extract was prepared by centrifugation at $15,000 \times g$ at $4^{\circ} \mathrm{C}$ for $45 \mathrm{~min}$. Protein extracts were quantified by the Pierce BCA protein assay (Thermo Fisher Scientific). Equal amounts of proteins were loaded, separated by $15 \%$ SDS-PAGE, and transferred to a nitrocellulose membrane (GE Healthcare), followed by immunoblotting analysis. Rabbit polyclonal antibodies raised against anti-FLAG (Sigma-Aldrich) or anti-6xHis (SigmaAldrich) were diluted to 1:5,000. Rabbit antibodies were detected with staphylococcal protein A conjugated to horseradish peroxidase (Sigma-Aldrich). As a loading control for these experiments, immunoblotting was carried out for the same 
protein extracts resolved on $15 \%$ acrylamide and probed with rabbit polyclonal antibodies raised against anti-GroEL (SigmaAldrich) diluted to 1:20,000. Anti-GroEL was detected with protein A conjugated to horseradish peroxidase (Sigma-Aldrich). Immunoblotting was developed with Pierce SuperSignal West Femto Chemiluminescent Substrate (Thermo Fisher Scientific) and exposed to Pierce CL-XPosure film (Thermo Fisher Scientific).

\section{One-hybrid system assay.}

For the transactivation assay, the flp3 promoter region ( -22 to -439) was inserted into EcoRI and SalI restriction sites of the plasmid pH3U3, which harbors a canonical Shine-Dalgarno sequence for $E$. coli. In addition, different transcriptional factors encoded by ' $\mathrm{Ca}$. L. asiaticus' were cloned downstream of the lacUV5 promoter and fused to the carboxi terminal of the E. coli RNA polymerase omega subunit and FLAG Tag into the

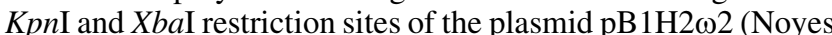
et al. 2008). All the constructs were used to cotransform the $E$. coli mutant strain $(\triangle r p o Z: z e o, \Delta h i s B, \Delta p y r F)$ and proceed with selection assays in minimal media without histidine plus 3aminotriazole and required antibiotics, as previously described (Noyes et al. 2008). To test the feasibility of this assay for ' $\mathrm{Ca}$. L. asiaticus', the first selection test was performed with the plasmid $\mathrm{pH} 3 \mathrm{U} 3$ harboring the $l d t P$ promoter region $(-15$ to -380) with its transcriptional regulator $l d t R$ cloned into $\mathrm{pB} 1 \mathrm{H} 2 \omega 2$, because the binding motif of $l d t R$ to the $l d t P$ promoter was previously well characterized in ' $\mathrm{Ca}$. L. asiaticus' (Pagliai et al. 2014 and 2017).

\section{$\beta$-Galactosidase assay.}

Fragments containing the promoter elements upstream of cpaA (CLIBASIA_03080), cpaF (CLIBASIA_03055), and flp3 (CLIBASIA_03105) were amplified from ' $\mathrm{Ca}$. L. asiaticus' genomic DNA isolated from the plant host using Phusion DNA polymerase and primers. PCR fragments were agarose gelpurified, ligated into pGEM-T Easy, and verified by sequencing. Fragments were excised with the appropriate restriction enzymes and ligated into the compatibly cleaved vector pRA301. Fragments were designed to insert the promoter region and ribosome-binding site with the start codon of the gene of interest in frame with the promoterless lac $Z$ gene. Fragments were confirmed by PCR, and the constructs were electroporated into A. tumefaciens wild-type and $\Delta v i s N R$ mutant strains. Bacterial cells were prepared for $\beta$-galactosidase assay by culturing in ATGN into the exponential phase measured for $\mathrm{OD}_{600}$ and frozen at $-80^{\circ} \mathrm{C}$. For $\beta$-galactosidase activity, cells were permeabilized in $\mathrm{Z}$ buffer $\left(60 \mathrm{mM} \mathrm{Na} \mathrm{HPO}_{4}, 40 \mathrm{mM} \mathrm{NaH} \mathrm{PO}_{4}\right.$, $10 \mathrm{mM} \mathrm{KCl}, 1 \mathrm{mM} \mathrm{MgSO}$, $50 \mathrm{mM} \beta$-mercaptoethanol, $\mathrm{pH} 7.0$ ) by the addition of three drops of $0.05 \%$ SDS and four drops of chloroform (Hibbing and Fuqua 2011). $\beta$ Galactosidase reactions were initiated by addition of $100 \mu \mathrm{l}$ of a colorimetric substrate $o$-nitrophenyl $\beta$-D-galactopyranoside (ONPG) solution concentration of $4 \mathrm{mg} / \mathrm{ml}$ and terminated with addition of $600 \mu \mathrm{l}$ of $6 \mathrm{M} \mathrm{NaCO}_{2}$. Intact cells and debris were removed by centrifugation $(10 \mathrm{~min}, 10,000 \times g)$, free ONP was measured as $A_{420}$, and specific activity was reported in Miller units. At least three biological replicates were performed.

\section{Pull-down assay.}

$E$. coli cells expressing ' $C a$. L. asiaticus' 6xHisVisR protein were washed in PBS and suspended in Lysis buffer (PBS, $20 \mathrm{mM}$ imidazole, $100 \mu \mathrm{g}$ of lysozyme per milliliter, $5 \mathrm{mM}$ $\mathrm{MgSO}_{4}, 40 \mu \mathrm{g}$ of DNAseI per milliliter, $\mathrm{pH}$ 7.4) and sonicated to generate the cell lysates. After centrifugation, the cell lysates were incubated with nickel-IMAC beads (Thermo Fisher Scientific). The beads were washed four times with PBS plus $20 \mathrm{mM}$ imidazole at $\mathrm{pH} 7.4$ to remove the unbound proteins and incubated with $E$. coli cell lysates containing ' $\mathrm{Ca}$. L. asiaticus' VisN-FLAG for $2 \mathrm{~h}$ at $4^{\circ} \mathrm{C}$. After washing four times, the beads were resuspended in SDS-PAGE loading buffer, followed by boiling for $5 \mathrm{~min}$. The samples are subjected to SDS-PAGE and immunoblotting using anti-His and anti-FLAG (SigmaAldrich) antibodies as described above.

\section{Protein in vivo stability assay.}

To test the in vivo stability of ' $\mathrm{Ca}$. L. asiaticus' VisN-Flag in the presence of ' $\mathrm{Ca}$. L. asiaticus' VisR, $10 \mathrm{ml}$ of overnight A. tumefaciens cultures coexpressing VisN-Flag with VisR or an empty vector, as a control, were pelleted and resuspended in $10 \mathrm{ml}$ of fresh medium for an additional $1 \mathrm{~h}$ of incubation. After incubation, spectinomycin solution was directly added to each culture at a final concentration of $1 \mathrm{mg} / \mathrm{ml}$ to block protein synthesis. A 1-ml aliquot was harvested at each time point and lysed in protein loading buffer followed by boiling for $5 \mathrm{~min}$. The samples were subjected to SDS-PAGE and immunoblotting. The SDS-PAGE gel was transferred to a nitrocellulose membrane (Millipore) using a Trans-Blot Turbo system (Bio-Rad Laboratories). The membrane was blocked using 5\% dry milk in TBST buffer (50 mM Tris, $150 \mathrm{mM} \mathrm{NaCl}, 0.05 \%$ Tween $20, \mathrm{pH}$ 7.6). The membrane was incubated with anti-FLAG and detected by protein A conjugated to horseradish peroxidase. Immunoactive proteins were detected using a ECL detection kit and X-ray film. Anti-GroEL antibodies were used to normalize the amount of protein loaded.

\section{Expression and purification of $6 \mathrm{HisVisR}$ in $E$. coli.}

For expression of the recombinant protein $6 \mathrm{HisVisR}$ in $E$. coli, a fragment containing the visR coding region sequence was amplified by PCR using ' $\mathrm{Ca}$. L. asiaticus' genomic DNA as template and the primers F-pETVisR and R-pETVisR. The amplified fragment was purified and digested with $\mathrm{Nde} \mathrm{I} /$ HindIII and inserted into pET-28a (Novagen), resulting in pET-6HisVisR. Recombinant protein 6HisVisR was overexpressed in E. coli BL21 Star (DE3) pLysS (Novagen) by addition of $1 \mathrm{mM}$ IPTG to cells grown to the midexponential phase at $37^{\circ} \mathrm{C}$ and $180 \mathrm{rpm}$. After addition of IPTG, the temperature was changed to $22^{\circ} \mathrm{C}$, and the $6 \mathrm{HisVisR}$ induction occurred overnight. Next, bacterial cells were harvested by centrifugation, and cell pellets were resuspended in $50 \mathrm{mM}$ $\mathrm{NaH}_{2} \mathrm{PO}_{4}(\mathrm{pH} 8.0), 300 \mathrm{mM} \mathrm{NaCl}, 10 \mathrm{mM}$ imidazole, and $1 \mathrm{mg}$ of lysozyme per milliliter. Cells were ruptured by sonication. Cell-free extract was prepared by centrifugation at $14,000 \times g$ at $4^{\circ} \mathrm{C}$ for $45 \mathrm{~min}$. The recombinant protein 6HisVisR was purified by Pierce Ni-NTA affinity chromatography (Thermo Fisher Scientific) according to the manufacturer recommendations. The protein-containing fractions were concentrated, and the buffer was exchanged with PBS buffer $\left(10 \mathrm{mM} \mathrm{Na} 2 \mathrm{HPO}_{4}, 2 \mathrm{mM} \mathrm{KH} \mathrm{KHO}_{4}, 2.7 \mathrm{mM} \mathrm{KCl}\right.$,

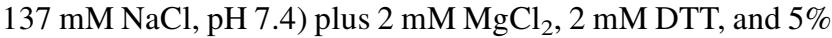
glycerol. The protein concentration was estimated using a Pierce BCA method (Thermo Fisher Scientific) with bovine serum albumin as the standard. The purified protein was stored at $-80^{\circ} \mathrm{C}$.

\section{EMSA.}

For EMSA, the recombinant protein $6 \mathrm{His} V i s \mathrm{R}$ was induced and purified as described above. Oligonucleotides Flp3p_Las-F and Flp3p_Las-R were used to amplify part of the flp3 promoter region $(-198$ to +1$)$ using PCR. The purified DNA fragment coding the flp3 promoter was labeled using a Pierce Biotin 3' End DNA Labeling Kit (Thermo Fisher Scientific). EMSA was conducted using a LightShift Chemiluminescent EMSA Kit (Thermo Fisher Scientific). The binding reactions with the labeled probe incubated with $100 \mathrm{nM} 6 \mathrm{His}$ VisR were resolved in a 
5\% nondenaturing polyacrylamide TBE gel. The EMSA was developed in accordance with the manufacturer instructions.

\section{ACKNOWLEDGMENTS}

We thank S. Gelvin (Purdue University) for sending us the strain A. tumefaciens C58 and J. Barrick (University of Texas-Austin) for giving us the pRA301 vector.

\section{LITERATURE CITED}

Akakura, R., and Winans, S. C. 2002. Mutations in the occQ operator that decrease OccR-induced DNA bending do not cause constitutive promoter activity. J. Biol. Chem. 277:15773-15780.

Ammar, E.-D., Ramos, J. E., Hall, D. G., Dawson, W. O., and Shatters, R. G., Jr. 2016. Acquisition, replication and inoculation of Candidatus Liberibacter asiaticus following various acquisition periods on huanglongbing-infected citrus by nymphs and adults of the Asian citrus psyllid. PLoS One 11:e0159594.

Andrade, M. O., Farah, C. S., and Wang, N. 2014. The post-transcriptional regulator $r s m A / c s r A$ activates T3SS by stabilizing the 5' UTR of $h r p G$, the master regulator of $h r p / h r c$ genes, in Xanthomonas. PLoS Pathog. 10: e1003945.

Angelov, A., Bergen, P., Nadler, F., Hornburg, P., Lichev, A., Übelacker, M. Pachl, F., Kuster, B., and Liebl, W. 2015. Novel Flp pilus biogenesisdependent natural transformation. Front. Microbiol. 6:84.

Avidan, O., Petrenko, M., Becker, R., Beck, S., Linscheid, M., Pietrokovski, S., and Jurkevitch, E. 2017. Identification and characterization of differentially-regulated type IVb pilin genes necessary for predation in obligate bacterial predators. Sci. Rep. 7:1013

Barnett, M. J., Fisher, R. F., Jones, T., Komp, C., Abola, A. P., BarloyHubler, F., Bowser, L., Capela, D., Galibert, F., Gouzy, J., Gurjal, M., Hong, A., Huizar, L., Hyman, R. W., Kahn, D., Kahn, M. L., Kalman, S., Keating, D. H., Palm, C., Peck, M. C., Surzycki, R., Wells, D. H., Yeh, K. C., Davis, R. W., Federspiel, N. A., and Long, S. R. 2001. Nucleotide sequence and predicted functions of the entire Sinorhizobium meliloti pSymA megaplasmid. Proc. Natl. Acad. Sci. U.S.A. 98:9883-9888.

Bendix, C., and Lewis, J. D. 2018. The enemy within: Phloem-limited pathogens. Mol. Plant Pathol. 19:238-254.

Bernard, C. S., Bordi, C., Termine, E., Filloux, A., and de Bentzmann, S. 2009 Organization and PprB-dependent control of the Pseudomonas aeruginosa tad locus, involved in Flp pilus biology. J. Bacteriol. 191:1961-1973.

Capela, D., Barloy-Hubler, F., Gouzy, J., Bothe, G., Ampe, F., Batut, J., Boistard, P., Becker, A., Boutry, M., Cadieu, E., Dréano, S., Gloux, S., Godrie, T., Goffeau, A., Kahn, D., Kiss, E., Lelaure, V., Masuy, D., Pohl, T., Portetelle, D., Pühler, A., Purnelle, B., Ramsperger, U., Renard, C., Thébault, P., Vandenbol, M., Weidner, S., and Galibert, F. 2001. Analysis of the chromosome sequence of the legume symbiont Sinorhizobium meliloti strain 1021. Proc. Natl. Acad. Sci. U.S.A. 98:9877-9882.

Christen, M., Beusch, C., Bösch, Y., Cerletti, D., Flores-Tinoco, C. E., Del Medico, L., Tschan, F., and Christen, B. 2016. Quantitative selection analysis of bacteriophage $\varphi \mathrm{CbK}$ susceptibility in Caulobacter crescentus. J. Mol. Biol. 428(2 Pt B):419-430.

Chung, I. Y., Jang, H. J., Bae, H. W., and Cho, Y. H. 2014. A phage protein that inhibits the bacterial ATPase required for type IV pilus assembly. Proc. Natl. Acad. Sci. U.S.A. 111:11503-11508.

Cicero, J. M., Fisher, T. W., and Brown, J. K. 2016. Localization of 'Candidatus Liberibacter solanacearum' and evidence for surface appendages in the potato psyllid vector. Phytopathology 106:142-154.

Cicero, J. M., Fisher, T. W., Qureshi, J. A., Stansly, P. A., and Brown, J. K. 2017. Colonization and intrusive invasion of potato psyllid by 'Candidatus Liberibacter solanacearum'. Phytopathology 107:36-49.

Clark, K., Franco, J. Y., Schwizer, S., Pang, Z., Hawara, E., Liebrand, T. W. H., Pagliaccia, D., Zeng, L., Gurung, F. B., Wang, P., Shi, J., Wang, Y., Ancona, V., van der Hoorn, R. A. L., Wang, N., Coaker, G., and Ma, W. 2018. An effector from the huanglongbing-associated pathogen targets citrus proteases. Nat. Commun. 9:1718.

Clock, S. A., Planet, P. J., Perez, B. A., and Figurski, D. H. 2008. Outer membrane components of the Tad (tight adherence) secreton of Aggregatibacter actinomycetemcomitans. J. Bacteriol. 190:980-990.

Coletta-Filho, H. D., Daugherty, M. P., Ferreira, C., and Lopes, J. R. 2014. Temporal progression of 'Candidatus Liberibacter asiaticus' infection in citrus and acquisition efficiency by Diaphorina citri. Phytopathology 104:416-421.

Costa, T. R. D., Ilangovan, A., Ukleja, M., Redzej, A., Santini, J. M., Smith, T. K., Egelman, E. H., and Waksman, G. 2016. Structure of the bacterial sex $F$ pilus reveals an assembly of a stoichiometric protein-phospholipid complex. Cell 166:1436-1444.E10.

de Bentzmann, S., Aurouze, M., Ball, G., and Filloux, A. 2006. FppA, a novel Pseudomonas aeruginosa prepilin peptidase involved in assembly of type IVb pili. J. Bacteriol. 188:4851-4860.

Duan, Y., Zhou, L., Hall, D. G., Li, W., Doddapaneni, H., Lin, H., Liu, L., Vahling, C. M., Gabriel, D. W., Williams, K. P., Dickerman, A., Sun, Y., and Gottwald, T. 2009. Complete genome sequence of citrus huanglongbing bacterium, 'Candidatus Liberibacter asiaticus' obtained through metagenomics. Mol. Plant-Microbe Interact. 22: 1011-1020.

Ellison, C. K., Kan, J., Dillard, R. S., Kysela, D. T., Ducret, A., Berne, C., Hampton, C. M., Ke, Z., Wright, E. R., Biais, N., Dalia, A. B., and Brun, Y. V. 2017. Obstruction of pilus retraction stimulates bacterial surface sensing. Science 358:535-538.

Ghanim, M., Fattah-Hosseini, S., Levy, A., and Cilia, M. 2016. Morphological abnormalities and cell death in the Asian citrus psyllid (Diaphorina citri) midgut associated with Candidatus Liberibacter asiaticus. Sci. Rep. 6:33418.

Hansen, J. K., and Forest, K. T. 2006. Type IV pilin structures: Insights on shared architecture, fiber assembly, receptor binding and type II secretion. J. Mol. Microbiol. Biotechnol. 11:192-207.

Hibbing, M. E., and Fuqua, C. 2011. Antiparallel and interlinked control of cellular iron levels by the Irr and RirA regulators of Agrobacterium tumefaciens. J. Bacteriol. 193:3461-3472.

Hibbing, M. E., and Fuqua, C. 2012. Inhibition and dispersal of Agrobacterium tumefaciens biofilms by a small diffusible Pseudomonas aeruginosa exoproduct(s). Arch. Microbiol. 194:391-403.

Hoang, H. H., Gurich, N., and González, J. E. 2008. Regulation of motility by the ExpR/Sin quorum-sensing system in Sinorhizobium meliloti J. Bacteriol. 190:861-871.

Kachlany, S. C., Planet, P. J., Desalle, R., Fine, D. H., Figurski, D. H., and Kaplan, J. B. 2001. flp-1, the first representative of a new pilin gene subfamily, is required for non-specific adherence of Actinobacillus actinomycetemcomitans. Mol. Microbiol. 40:542-554.

Kawahara, K., Oki, H., Fukakusa, S., Yoshida, T., Imai, T., Maruno, T., Kobayashi, Y., Motooka, D., Iida, T., Ohkubo, T., and Nakamura, S 2016. Homo-trimeric structure of the type IVb minor pilin CofB suggests mechanism of CFA/III pilus assembly in human enterotoxigenic Escherichia coli. J. Mol. Biol. 428:1209-1226.

Kruse, A., Fattah-Hosseini, S., Saha, S., Johnson, R., Warwick, E. R., Sturgeon, K., Mueller, L., MacCoss, M. J., Shatters, R. G., Jr., and Heck, M. C. 2017 Combining 'omics and microscopy to visualize interactions between the Asian citrus psyllid vector and the huanglongbing pathogen Candidatus Liberibacter asiaticus in the insect gut. PLoS One 12:e0179531.

Kuykendall, L. D., Shao, J. Y., and Hartung, J. S. 2012. Conservation of gene order and content in the circular chromosomes of 'Candidatus Liberibacter asiaticus' and other Rhizobiales. PLoS One 7:e34673.

Leighton, T. L., Buensuceso, R. N., Howell, P. L., and Burrows, L. L. 2015. Biogenesis of Pseudomonas aeruginosa type IV pili and regulation of their function. Environ. Microbiol. 17:4148-4163.

Li, J., Li, L., Pang, Z., Kolbasov, V. G., Ehsani, R., Carter, E. W., and Wang, N. 2019. Developing citrus huanglongbing (HLB) management strategies based on the severity of symptoms in HLB-endemic citrusproducing regions. Phytopathology 109:582-592.

Li, J., and Wang, N. 2011. Genome-wide mutagenesis of Xanthomonas axonopodis pv. citri reveals novel genetic determinants and regulation mechanisms of biofilm formation. PLoS One 6:e21804.

Li, T., Xu, Z., Zhang, T., Li, L., Chen, H., and Zhou, R. 2012. The genetic analysis of the flp locus of Actinobacillus pleuropneumoniae. Arch. Microbiol. 194:167-176.

Livak, K. J., and Schmittgen, T. D. 2001. Analysis of relative gene expression data using real-time quantitative PCR and the $2^{-\Delta \Delta C T}$ method. Methods 25:402-408.

Mendoza-Herrera, A., Levy, J., Harrison, K., Yao, J., Ibanez, F., and Tamborindeguy, C. 2018. Infection by 'Candidatus Liberibacter solanacearum' haplotypes A and B in Solanum lycopersicum 'Moneymaker'. Plant Dis. 102:2009-2015.

Mignolet, J., Panis, G., and Viollier, P. H. 2018. More than a Tad: Spatiotemporal control of Caulobacter pili. Curr. Opin. Microbiol. 42:79-86

Morton, E. R., and Fuqua, C. 2012. Laboratory maintenance of Agrobacterium. Curr. Protoc. Microbiol. 24:3D.1.1-3D.1.6.

Noyes, M. B., Meng, X., Wakabayashi, A., Sinha, S., Brodsky, M. H., and Wolfe, S. A. 2008. A systematic characterization of factors that regulate Drosophila segmentation via a bacterial one-hybrid system. Nucleic Acids Res. 36:2547-2560.

Nykyri, J., Mattinen, L., Niemi, O., Adhikari, S., Kõiv, V., Somervuo, P., Fang, X., Auvinen, P., Mäe, A., Palva, E. T., and Pirhonen, M. 2013. Role 
and regulation of the Flp/Tad pilus in the virulence of Pectobacterium atrosepticum SCRI1043 and Pectobacterium wasabiae SCC3193. PLoS One 8:e73718.

O’Connell Motherway, M., Zomer, A., Leahy, S. C., Reunanen, J., Bottacini, F., Claesson, M. J., O’Brien, F., Flynn, K., Casey, P. G., Munoz, J. A., Kearney, B., Houston, A. M., O’Mahony, C., Higgins, D. G., Shanahan, F., Palva, A., de Vos, W. M., Fitzgerald, G. F., Ventura, M., O'Toole, P. W., and van Sinderen, D. 2011. Functional genome analysis of Bifidobacterium breve UCC2003 reveals type IVb tight adherence (Tad) pili as an essential and conserved host-colonization factor. Proc. Natl. Acad. Sci. U.S.A. 108:11217-11222.

Pagliai, F. A., Coyle, J. F., Kapoor, S., Gonzalez, C. F., and Lorca, G. L. 2017. LdtR is a master regulator of gene expression in Liberibacter asiaticus. Microb. Biotechnol. 10:896-909.

Pagliai, F. A., Gardner, C. L., Bojilova, L., Sarnegrim, A., Tamayo, C., Potts, A. H., Teplitski, M., Folimonova, S. Y., Gonzalez, C. F., and Lorca, G. L. 2014. The transcriptional activator LdtR from 'Candidatus Liberibacter asiaticus' mediates osmotic stress tolerance. PLoS Pathog. 10:e1004101

Rotter, C., Mühlbacher, S., Salamon, D., Schmitt, R., and Scharf, B. 2006. Rem, a new transcriptional activator of motility and chemotaxis in Sinorhizobium meliloti. J. Bacteriol. 188:6932-6942.

Roux, N., Spagnolo, J., and de Bentzmann, S. 2012. Neglected but amazingly diverse type IVb pili. Res. Microbiol. 163:659-673.

Sambrook, J., and Russell, D. W. 2006. Directional cloning into plasmid vectors. Cold Spring Harb. Protoc. 2006:e3919.

Sétamou, M., Alabi, O. J., Kunta, M., Jifon, J. L., and da Graça, J. V. 2016. Enhanced acquisition rates of 'Candidatus Liberibacter asiaticus' by the Asian citrus psyllid (Hemiptera: Liviidae) in the presence of vegetative flush growth in citrus. J. Econ. Entomol. 109:1973-1978.

Sourjik, V., Muschler, P., Scharf, B., and Schmitt, R. 2000. VisN and VisR are global regulators of chemotaxis, flagellar, and motility genes in Sinorhizobium (Rhizobium) meliloti. J. Bacteriol. 182:782-788.

Tambalo, D. D., Del Bel, K. L., Bustard, D. E., Greenwood, P. R., Steedman, A. E., and Hynes, M. F. 2010. Regulation of flagellar, motility and chemotaxis genes in Rhizobium leguminosarum by the VisN/R-Rem cascade. Microbiology 156:1673-1685.

Thompson, M. A., Onyeziri, M. C., and Fuqua, C. 2018. Function and regulation of Agrobacterium tumefaciens cell surface structures that promote attachment. Pages 143-184 in: Agrobacterium Biology: From Basic Science to Biotechnology. B. Gelvin, ed. Current Topics in Microbiology and Immunology, vol. 418. Springer Nature, Basingstoke, U.K.

Tomich, M., Fine, D. H., and Figurski, D. H. 2006. The TadV protein of Actinobacillus actinomycetemcomitans is a novel aspartic acid prepilin peptidase required for maturation of the Flp1 pilin and TadE and TadF pseudopilins. J. Bacteriol. 188:6899-6914.

Tomich, M., Planet, P. J., and Figurski, D. H. 2007. The tad locus: Postcards from the widespread colonization island. Nat. Rev. Microbiol. 5: 363-375.

Wairuri, C. K., van der Waals, J. E., van Schalkwyk, A., and Theron, J 2012. Ralstonia solanacearum needs Flp pili for virulence on potato. Mol. Plant-Microbe Interact. 25:546-556.

Wang, J., Haapalainen, M., Schott, T., Thompson, S. M., Smith, G. R., Nissinen, A. I., and Pirhonen, M. 2017a. Genomic sequence of 'Candidatus Liberibacter solanacearum' haplotype $\mathrm{C}$ and its comparison with haplotype A and B genomes. PLoS One 12:e0171531.

Wang, N., Pierson, E. A., Setubal, J. C., Xu, J., Levy, J. G., Zhang, Y., Li, J., Rangel, L. T., and Martins, J., Jr. 2017b. The Candidatus Liberibacterhost interface: Insights into pathogenesis mechanisms and disease control. Annu. Rev. Phytopathol. 55:451-482.

Wang, N., and Trivedi, P. 2013. Citrus huanglongbing: A newly relevant disease presents unprecedented challenges. Phytopathology 103: 652-665.

Wang, Y., Haitjema, C. H., and Fuqua, C. 2014. The Ctp type IVb pilus locus of Agrobacterium tumefaciens directs formation of the common pili and contributes to reversible surface attachment. J. Bacteriol. 196: 2979-2988.

Weigel, D., and Glazebrook, J. 2006. Transformation of Agrobacterium using electroporation. Cold Spring Harb. Protoc. 2006:e4665.

Wise, A. A., Liu, Z., and Binns, A. N. 2006. Culture and maintenance of Agrobacterium strains. Methods Mol. Biol. 343:3-13.

Wulff, N. A., Zhang, S., Setubal, J. C., Almeida, N. F., Martins, E. C., Harakava, R., Kumar, D., Rangel, L. T., Foissac, X., Bové, J. M., and Gabriel, D. W. 2014. The complete genome sequence of 'Candidatus Liberibacter americanus', associated with citrus huanglongbing. Mol. Plant-Microbe Interact. 27:163-176.

Xu, J., Kim, J., Koestler, B. J., Choi, J. H., Waters, C. M., and Fuqua, C. 2013. Genetic analysis of Agrobacterium tumefaciens unipolar polysaccharide production reveals complex integrated control of the motileto-sessile switch. Mol. Microbiol. 89:929-948.

Yan, Q., Sreedharan, A., Wei, S., Wang, J., Pelz-Stelinski, K., Folimonova, S., and Wang, N. 2013. Global gene expression changes in Candidatus Liberibacter asiaticus during the transmission in distinct hosts between plant and insect. Mol. Plant Pathol. 14:391-404.

Zatakia, H. M., Nelson, C. E., Syed, U. J., and Scharf, B. E. 2014. ExpR coordinates the expression of symbiotically important, bundle-forming Flp pili with quorum sensing in Sinorhizobium meliloti. Appl. Environ. Microbiol. 80:2429-2439. 